\title{
Entropy Based Risk Measures
}

\author{
Alois Pichler* $\quad$ Ruben Schlotter ${ }^{\dagger}$
}

January 23, 2018

\begin{abstract}
Entropy is a measure of self-information which is used to quantify information losses. Entropy was developed in thermodynamics, but is also used to compare probabilities based on their deviating information content. Corresponding model uncertainty is of particular interest and importance in stochastic programming and its applications like mathematical finance, as complete information is not accessible or manageable in general.

This paper extends and generalizes the Entropic Value-at-Risk by involving Rényi entropies. We provide explicit relations of different entropic risk measures, we elaborate their dual representations and present their relations explicitly.

We consider the largest spaces which allow studying the impact of information in detail and it is demonstrated that these do not depend on the information loss. The dual norms and Hahn-Banach functionals are characterized explicitly.

Keywords: Risk Measures, Rearrangement Inequalities, Stochastic Dominance, Dual Representation

Classification: 90C15, 60B05, 62P05
\end{abstract}

\section{Introduction}

Boltzmann (1877) defines the entropy of a thermodynamic system as a measure of how many different microstates could give rise to the macrostate the system is in. He gives the famous formula

$$
S=k \log W
$$

for the entropy of the thermodynamic system, where $S$ is the entropy of the macrostate, $k$ is Boltzmann's constant and $W$ is the total number of possible microstates that might yield the macrostate. It then becomes natural to interpret entropy as a measure of disorder. Shannon (1948) defines the information entropy of a discrete random variable $Z$ with probability mass function $f(\cdot)$ by

$$
H(Z)=\sum_{x} f(x) \log f(x)
$$

which extends to

$$
H(Z)=\mathbb{E} Z \log Z
$$

*Both authors: Technische Universität Chemnitz, Fakultät für Mathematik. 90126 Chemnitz, Germany.

$\dagger$ Corresponding author. Contact: ruben.schlotter@math.tu-chemnitz.de 
in the continuous case. Information entropy is interpreted as the average amount of information produced by the probabilistic source of data $Z$. Relating this to Boltzmann's entropy formula one can say that information entropy of a system is the amount of information needed to determine a microstate, given the macrostate. Many extensions of information entropy (now often called Shannon entropy) have been introduced. The most notable extensions are Rényi entropies of order q, specified as

$$
H_{q}(Z):=\frac{1}{q-1} \log \mathbb{E} Z^{q}
$$

Related to Shannon entropy is the quantity

$$
D(Q \| P)=\sum_{x} f(x) \log \frac{f(x)}{g(x)}
$$

called relative entropy or Kullback-Leibler divergence. Here, $f(\cdot)(g(\cdot)$, resp.) is the probability mass functions of the probability measure $P(Q$, resp.). Relative entropy describes the information loss when considering the distribution $g$ while $f$ is the correct distribution. Divergence can therefore be seen as a distance of probability measures, although it is not a metric since it is neither symmetric nor does it satisfy the triangle inequality. Divergences can be derived from different entropies in analogy to relative entropy. For Rényi entropy we obtain Rényi divergences given by

$$
D_{R}^{q}(Q \| P)=\frac{1}{q-1} \log \mathbb{E} Z^{q}=H_{q}(Z),
$$

where $Z=\frac{\mathrm{d} Q}{\mathrm{~d} P}$ is the Radon-Nikodým derivative. The family of Rényi divergences is related to Hellinger divergences defined as

$$
D_{T}^{q}(Q \| P)=\frac{1}{q-1} \mathbb{E}\left(Z^{q}-1\right)
$$

(see Liese and Vajda (2006)), where $Z$ is as above. Hellinger divergence is sometimes called Tsallis divergence.

For an overview of entropy in information theory we may refer to Cover and Thomas (2006). For the relationship between different divergences see Cichocki and Amari (2010), Amari (2009) and Liese and Vajda (2006). For information specific to Rényi divergence we refer the interested reader to van Erven and Harremos (2014).

Risk measures play an important role in finance, stochastic optimization, e.g. In fact, in risk-averse stochastic optimization one is often interested in problems of the form

$$
\begin{aligned}
& \operatorname{minimize} \rho(c(x, Y)) \\
& \text { subject to } x \in \mathbb{X}
\end{aligned}
$$

where $\rho$ is a risk measure, $c$ is a cost function of a random variable $Y$ and $\mathbb{X}$ is a decision space. In this paper we focus our attention on risk measures based on entropy. In particular, we address generalizations of the Entropic Value-at-Risk (EV@R), a coherent risk measure introduced in Ahmadi-Javid (2012a,b). It is known that EV@R can be represented using relative entropy or Kullback-Leibler divergence as

$$
\mathrm{EV} @ \mathrm{R}_{\alpha}(Y)=\sup \left\{\mathbb{E} Y Z: Z \geq 0, \mathbb{E} Z=1, \mathbb{E} Z \log Z \leq \log \frac{1}{1-\alpha}\right\},
$$


where $Z$ is a density with respect to the reference probability measure $P$. This risk measure corresponds to the worst expected value with respect to probability measures with information content not deviating by more than $\log \frac{1}{1-\alpha}$ from the baseline distribution $P$.

In this paper we generalize the risk measure (2) and consider new risk measures by replacing the relative entropy in the dual representation (2) with different divergences as suggested in Ahmadi-Javid (2012a) first. Breuer and Csiszár (2013a,b) study the class of $\phi$-entropic risk measures (cf. also Bellini and Rosazza Gianin (2008)). Kovacevic and Breuer (2016) study a multiperiod extension of (2) while Föllmer and Knispel (2011) study a version of an entropic risk measure too, but in a very different conceptual setting.

Extending this direction of research involving information losses motivates studying risk measures of the form

$$
\rho(Y)=\sup \left\{\mathbb{E} Y Z: Z \geq 0, \mathbb{E} Z=1, H_{q}(Z) \leq \log \frac{1}{1-\alpha}\right\},
$$

where $H_{q}$ is the Rényi entropy. For $q>1$ these risk measures are related to higher order dual risk measures considered in Dentcheva et al. (2010). Incorporating entropy into the definition of the risk measure allows a consistent interpretation based on information theory.

The new class of risk measures based on Rényi divergence recovers well-known coherent risk measures such as the Average Value-at-risk (AV@R), the expectation and the essential supremum, the classical Entropic Value-at-Risk is a special case as well. In fact, our new class of entropy based risk measures interpolates the Average Value-at-Risk and the classical Entropic Value-at-Risk. This provides a flexible class of risk measures for modeling stochastic optimization problems taking information into account.

Returning to the standard problem (1) in risk-averse stochastic optimization we notice that for risk measures of the form (3) the problem (1) becomes a minimax problem which in general is difficult. However, extending the work of Dentcheva et al. (2010) we provide an equivalent infimum representation of (3) which facilitates the problem.

We further study the norms associated with the risk measure $\rho$ defined as

$$
\|Y\|_{\rho}:=\rho(|Y|)
$$

and their corresponding dual norms. We give explicit characterizations for the associated Hahn-Banach functionals.

Mathematical Setting. We consider a vector space $L$ of $\mathbb{R}$-valued random variables on a reference probability space $(\Omega, \mathcal{F}, P)$. The set $L$ is called model space, which is used in this paper to represent a set of random losses. This setting is typical in stochastic optimization as well as in the insurance literature, while in a finance context one is primarily interested in monetary gains instead of losses. Throughout this paper we work with random variables in $L^{p}(\Omega, \mathcal{F}, P)$ for $p \geq 1$ or $L^{\infty}(\Omega, \mathcal{F}, P)$. We shall call exponents $p$ and $q$ conjugate (Hölder conjugate, resp.), if $1 / p+1 / q=1$, even for $p<1$ or $q<1$. Typically, we denote the exponent conjugate to $p$ by $p^{\prime}$. As usual, we set $p^{\prime}=\infty$ for $p=1$ and $p^{\prime}=1$ for $p=\infty$.

A risk measure $\rho: L \rightarrow \mathbb{R} \cup\{\infty\}$ is called coherent if it satisfies the following four properties introduced by Artzner et al. (1999).

(i) Translation equivariance: $\rho(Y+c)=\rho(Y)+c$ for any $Y \in L$ and $c \in \mathbb{R}$;

(ii) Subadditivity: $\rho\left(Y_{1}+Y_{2}\right) \leq \rho\left(Y_{1}\right)+\rho\left(Y_{2}\right)$ for all $Y_{1}, Y_{2} \in L$;

(iii) Monotonicity: if $Y_{1}, Y_{2} \in L$ and $Y_{1} \leq Y_{2}$, then $\rho\left(Y_{1}\right) \leq \rho\left(Y_{2}\right)$;

(iv) Positive homogeneity: $\rho(\lambda Y)=\lambda \rho(Y)$ for all $Y \in L$ and $\lambda>0$. 
Delbaen (2002) gives a general representation of coherent risk measures in the form

$$
\rho(Y)=\sup \left\{\mathbb{E}_{Q} Y: Q \in \Gamma\right\},
$$

where $\Gamma$ is a set of probability measures satisfying certain regularity conditions. Risk measures considered in (3) are thus coherent.

Outline of the paper. Section 2 introduces and discusses important properties of the Rényi entropy, Section 3 then introduces the new risk measure EV@R and discusses its domain. In Section 4 we give the dual representation while Section 5 relates EV@R-norms with the norm in $L^{p}$. This section discusses the relation of different levels and orders as well. Section 6 deals with the dual norms and the Kusuoka representation, Section 7 concludes.

\section{Entropy}

This section defines the Rényi entropy and features important properties which we relate to in later sections.

Definition 1. We shall call a random variable $Z \in L^{1}$ with $Z \geq 0$ and $\mathbb{E} Z=1$ a density with respect to $P$, or simply density.

Definition 2. The Rényi entropy ${ }^{1}$ of order $q \in \mathbb{R}$ of a density $Z$ is $^{2}$

$$
H_{q}(Z):= \begin{cases}-\log P(Z>0) & \text { if } q=0, \\ \mathbb{E} Z \log Z & \text { if } q=1, \\ \log \|Z\|_{\infty} & \text { if } q=\infty, \\ \frac{1}{q-1} \log \mathbb{E} Z^{q} & \text { else, }\end{cases}
$$

provided that the expectations are finite (note that one has to assume $Z>0$ in order to have $H_{q}(Z)$ well-defined for $q<0)$.

Rényi entropy, as introduced in (6), is continuous in $q \in \mathbb{R}$. Indeed, by l'Hôpital's rule we have

$$
\lim _{q \rightarrow 1} H_{q}(Z)=\lim _{q \rightarrow 1} \frac{\mathbb{E} Z^{q} \log Z}{\mathbb{E} Z^{q}}=\mathbb{E} Z \log Z=H_{1}(Z),
$$

so that the entropy of order $q=1$ in (6) is the continuous extension of $\frac{1}{q-1} \log \mathbb{E} Z^{q}$. Furthermore, for $q \rightarrow \infty$, it holds that

$$
\lim _{q \rightarrow \infty} H_{q}(Z)=\lim _{q \rightarrow \infty} \frac{q}{q-1} \log \|Z\|_{q}=\log \|Z\|_{\infty} .
$$

For $q \rightarrow 0$ we get

$$
\lim _{q \rightarrow 0} H_{q}(Z)=\lim _{q \rightarrow 0} \frac{1}{q-1} \log \int_{\Omega} \mathbb{1}_{Z>0} Z^{q} \mathrm{~d} P=-\log P\{Z>0\}
$$

and hence the case $q=0$ in Definition 2 is consistent as well.

${ }^{1}$ Named after Alfréd Rényi, 1921-1970, Hungarian mathematician

${ }^{2}$ We employ the analytic continuation of the mapping $z \mapsto z \cdot \log z$ by setting $0 \log 0:=0$. 
Remark 3. The expression

$$
\|Z\|_{q}=\left(\mathbb{E} Z^{q}\right)^{\frac{1}{q}}
$$

is not a norm whenever $q<1$, but we will employ it to allow for a compact notation. With this notation at hand the Rényi entropy rewrites as

$$
H_{q}(Z)=\frac{q}{q-1} \log \|Z\|_{q}
$$

\section{Properties of Rényi Entropy}

The entropy $H_{q}(Z)$ is nonnegative for $q \geq 1$ as we have that $\|Z\|_{q} \geq \mathbb{E} Z=1$. For $0<q<1$ the exponents $\frac{1}{q}$ and $\frac{1}{1-q}$ are conjugate so that by Hölder's inequality $\mathbb{E} Z^{q} \leq\left\|Z^{q}\right\|_{\frac{1}{q}} \cdot\|\mathbb{1}\|_{\frac{1}{1-q}}=\mathbb{E} Z=1$ and consequently $H_{q}(Z)=\frac{1}{q-1} \log \mathbb{E} Z^{q} \geq 0$ even for $q \in(0,1)$. Together with the special case $q=0$ we thus have that the entropy is nonnegative for all $q \geq 0$,

$$
H_{q}(Z) \geq 0 \quad(q \geq 0)
$$

The elementary relation $P(Z>0) \cdot\|Z\|_{\infty} \geq 1$ follows from $\mathbb{E} Z=1$ and consequently we have that $H_{0}(Z) \leq H_{\infty}(Z)$. The next lemma reveals the general monotonic behavior of the Rényi entropy in its order $q$.

Lemma 4. The Rényi entropy $H_{q}(Z)$ is non-decreasing in its order $q$ for every $Z$ fixed. Further, there exists a density $Z$ with arbitrary entropy so that $H_{q}(Z)$ is constant for $q \geq 0$.

Proof. The derivative of (6) with respect to the order $q$ is

$$
\frac{\mathrm{d}}{\mathrm{d} q} H_{q}(Z)=-\frac{1}{(q-1)^{2}} \log \mathbb{E} Z^{q}+\frac{1}{q-1} \frac{\mathbb{E} Z^{q} \log Z}{\mathbb{E} Z^{q}},
$$

which can be restated as

$$
\begin{aligned}
\frac{\mathrm{d}}{\mathrm{d} q} H_{q}(Z) & =-\frac{1}{(q-1)^{2}} \log \mathbb{E} Z^{q}+\frac{q-1}{(q-1)^{2}} \mathbb{E} \frac{Z^{q}}{\mathbb{E} Z^{q}} \log Z \\
& =\frac{1}{(q-1)^{2}} \mathbb{E} \frac{Z^{q}}{\mathbb{E} Z^{q}} \log \frac{1}{Z} \frac{Z^{q}}{\mathbb{E} Z^{q}}=\frac{1}{(q-1)^{2}} \mathbb{E} Z_{q} \log \frac{Z_{q}}{Z},
\end{aligned}
$$

where we employ the abbreviation $Z_{q}$ for the power-density $Z_{q}:=\frac{Z^{q}}{\mathbb{E} Z^{q}}$. In line with the proof that the Kullback-Leibler divergence is non-negative we consider the Bregman divergence

$$
D(y, z):=\varphi(y)-\varphi(z)-\varphi^{\prime}(z) \cdot(y-z) .
$$

For the convex function $\varphi(z):=z \log z$ (with derivative $\varphi^{\prime}(z)=1+\log z$ ) we get by convexity

$$
0 \leq D\left(Z_{q}, Z\right)=Z_{q} \log Z_{q}-Z \log Z-(1+\log Z) \cdot\left(Z_{q}-Z\right) .
$$

Taking expectations and expanding gives

$$
\mathbb{E} Z_{q} \log \frac{Z_{q}}{Z}=\mathbb{E}\left[Z_{q} \log Z_{q}-Z \log Z-Z_{q} \log Z+Z \log Z\right] \geq 0 .
$$

It follows from (9) that $\frac{\mathrm{d}}{\mathrm{d} q} H_{q}(Z) \geq 0$ and thus the assertion. 
Now consider the random variable $Z$ with $\alpha \in(0,1)$ and $P(Z=0)=\alpha$ and $P\left(Z=\frac{1}{1-\alpha}\right)=1-\alpha$. The random variable $Z$ is a density with entropy

$$
H_{q}(Z)=\frac{1}{q-1} \log \frac{1-\alpha}{(1-\alpha)^{q}}=\log \frac{1}{1-\alpha},
$$

which is independent of the order $q$.

Remark 5. From Remark 3 it is clear that we have $H_{0}(Z)=0$ for $Z>0$ and hence Lemma 4 implies that

$$
H_{q}(Z) \leq 0
$$

for $q<0$.

We state convexity properties of the Rényi entropy for varying order $q$ next.

Proposition 6 (Convexity). The mapping $q \mapsto(q-1) \cdot H_{q}(Z)$ is a convex function on $\mathbb{R}$.

Proof. For $\lambda \in(0,1)$ and $q_{0}, q_{1} \in \mathbb{R}$ define $q_{\lambda}:=(1-\lambda) q_{0}+\lambda q_{1}$. By Hölder's inequality we have for arbitrary $q$ (such that every integral exists)

$$
\log \mathbb{E} Z^{q_{\lambda}}=\log \mathbb{E}\left(Z^{q} \cdot Z^{q_{\lambda}-q}\right) \leq \frac{1}{p} \log \left(\mathbb{E} Z^{q p}\right)+\frac{1}{p^{\prime}} \log \left(\mathbb{E} Z^{\left(q_{\lambda}-q\right) p^{\prime}}\right),
$$

where $p^{\prime}=\frac{p}{p-1}$ is Hölder's conjugate exponent to $p$.

Choose $p:=\frac{1}{1-\lambda}$ and $q:=(1-\lambda) q_{0}$ and observe that $q p=q_{0}, p^{\prime}=\frac{1}{\lambda}$ and $\left(q_{\lambda}-q\right) p^{\prime}=q_{1}$. The inequality (10) thus reads

$$
\log \mathbb{E} Z^{q_{\lambda}} \leq(1-\lambda) \log \left(\mathbb{E} Z^{q_{0}}\right)+\lambda \log \left(\mathbb{E} Z^{q_{1}}\right),
$$

from which the assertion follows.

The preceding Proposition 6 extends to the case $q=\infty$ in the following way.

Proposition 7. For $q, \tilde{q} \in \mathbb{R}$ and $q<\tilde{q}$ it holds that

$$
(\tilde{q}-1) H_{\tilde{q}}(Z) \leq(q-1) H_{q}(Z)+(\tilde{q}-q) H_{\infty}(Z)
$$

whenever the integrals are well defined.

Proof. Again by Hölder's inequality we have for $q<\tilde{q}$ that $\mathbb{E} Z^{\tilde{q}} \leq \mathbb{E}\left(Z^{q} \cdot\|Z\|_{\infty}^{\tilde{q}-q}\right)$. Thus

$$
\log \mathbb{E} Z^{\tilde{q}} \leq \log \mathbb{E} Z^{q}+(\tilde{q}-q) \log \|Z\|_{\infty},
$$

i.e.,

$$
(\tilde{q}-1) H_{\tilde{q}}(Z) \leq(q-1) H_{q}(Z)+(\tilde{q}-q) H_{\infty}(Z)
$$

which is the assertion. 


\section{Risk measures based on Rényi entropy}

We now define entropic risk measures based on Rényi entropy. We start from the dual representation of coherent risk measures first introduced in Delbaen (2002). The constant $\log \frac{1}{1-\alpha}$ in the definition below is chosen to relate the entropic risk measures to the Average Value-at-Risk and to the Value-at-Risk with confidence level $\alpha$.

Definition 8 (Risk measures based on Rényi entropy). The Entropic Value-at-Risk EV@ $\mathrm{R}_{\alpha}^{p}$ of order $p \in \mathbb{R}$ at confidence level $\alpha \in[0,1)$ and $Y \in L^{p}$ based on Rényi entropy is

$$
\mathrm{EV} @ \mathrm{R}_{\alpha}^{p}(Y):=\sup \left\{\mathbb{E} Y Z: Z \geq 0, \mathbb{E} Z=1 \text { and } H_{p^{\prime}}(Z) \leq \log \frac{1}{1-\alpha}\right\},
$$

where $\frac{1}{p}+\frac{1}{p^{\prime}}=1$. For $p=\infty$ we set EV@ $\mathrm{R}_{\alpha}^{\infty}(Y):=\operatorname{EV} @ \mathrm{R}_{\alpha}(Y)(\mathrm{cf} .(7))$, i.e.,

$$
\mathrm{EV} @ \mathrm{R}_{\alpha}(Y):=\sup \left\{\mathbb{E} Y Z: Z \geq 0, \mathbb{E} Z=1, \mathbb{E} Z \log Z \leq \log \frac{1}{1-\alpha}\right\}
$$

and for $p=1$

$$
\mathrm{EV} @ \mathrm{R}_{\alpha}^{1}(Y)=\operatorname{AV} @ \mathrm{R}_{\alpha}(Y):=\sup \left\{\mathbb{E} Y Z: Z \geq 0, \mathbb{E} Z=1, Z \leq \frac{1}{1-\alpha}\right\}
$$

For $\alpha=1$ we set EV@ $\mathrm{R}_{1}^{p}(Y):=\operatorname{ess} \sup Y$.

Remark 9 (The confidence level $\alpha=0$ ). The Entropic Value-at-Risk based on Rényi entropy is nondecreasing in $\alpha$, as $\alpha \mapsto \log \frac{1}{1-\alpha}$ is an increasing function. Also note that $\log \frac{1}{1-\alpha}=0$ whenever $\alpha=0$, and $H_{p^{\prime}}(Z)=0$

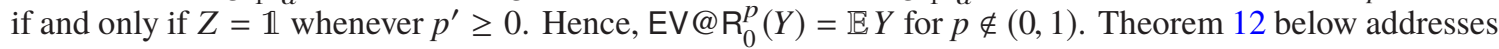
the case $p \in(0,1)$.

For $p>1$, the risk measure $\mathrm{EV} @ \mathrm{R}_{\alpha}^{p}(\cdot)$ is well defined on $L^{p}$ since

$$
\mathrm{EV}_{\mathrm{N}} \mathrm{R}_{\alpha}^{p}(Y) \leq\|Y\|_{p}\left(\frac{1}{1-\alpha}\right)^{\frac{1}{p}}
$$

An in-depth discussion of this case can be found in Dentcheva et al. (2010).

This paper particularly extends the Entropic Value-at-Risk for $p<1$. To this end it is useful to revise the Hölder and Minkowski inequality for $p<1$. Since the inequalities in both cases are reversed, they are sometimes called reverse Hölder and reverse Minkowski inequality, respectively.

Lemma 10 (Reverse Hölder and reverse Minkowski inequality). For $p \in(0,1)$ and $Y, Z \in L^{\infty}$ with $Z>0$ the inequality

$$
\|Y Z\|_{1} \geq\|Y\|_{p}\|Z\|_{q}
$$

holds true, where $q=\frac{p}{p-1}<0$ is the Hölder exponent conjugate to $p$.

For $Z_{1}, Z_{2} \in L^{\infty}$ such that $Z_{1}, Z_{2}>0$ and $q<1$ we have

$$
\left\|Z_{1}+Z_{2}\right\|_{q} \geq\left\|Z_{1}\right\|_{q}+\left\|Z_{2}\right\|_{q}
$$


Proof. Without loss of generality we may rescale $Y$ and $Z$ such that $\|Y Z\|_{1}=\|Z\|_{q}=1$. Then the desired inequality (14) reduces to $1 \geq\|Y\|_{p}^{p}=\left\|\left(\frac{|Y| Z}{Z}\right)^{p}\right\|_{1}$. To accept the latter apply Hölder's inequality to $\left(\frac{|Y| Z}{Z}\right)^{p}$ with $\frac{1}{p}>1$ and its conjugate Hölder exponent $\frac{1}{1-p}$, giving

$$
\left\|\left(\frac{|Y| Z}{Z}\right)^{p}\right\|_{1} \leq\left\|(|Y| Z)^{p}\right\|_{\frac{1}{p}} \cdot\left\|\frac{1}{Z^{p}}\right\|_{\frac{1}{1-p}}=\||Y| Z\|_{1}^{p} \cdot\|Z\|_{q}^{-p}=1
$$

and thus the statement.

We now derive the reverse Minkowski inequality by employing the reverse Hölder inequality. Let $Z_{1}, Z_{2} \in L^{\infty}$ be positive, then

$$
\left\|Z_{1}+Z_{2}\right\|_{q}^{q}=\mathbb{E} Z_{1}\left(Z_{1}+Z_{2}\right)^{q-1}+\mathbb{E} Z_{2}\left(Z_{1}+Z_{2}\right)^{q-1} .
$$

An application of the reverse Hölder inequality with conjugate exponents $q$ and $\frac{q}{q-1}$ gives

$$
\begin{aligned}
\mathbb{E} Z_{1}\left(Z_{1}+Z_{2}\right)^{q-1}+\mathbb{E} Z_{2}\left(Z_{1}+Z_{2}\right)^{q-1} & \geq\left(\left\|Z_{1}\right\|_{q}+\left\|Z_{2}\right\|_{q}\right)\left\|\left(Z_{1}+Z_{2}\right)^{q-1}\right\|_{\frac{q}{q-1}} \\
& =\left(\left\|Z_{1}\right\|_{q}+\left\|Z_{2}\right\|_{q}\right)\left(\mathbb{E}\left(Z_{1}+Z_{2}\right)^{q}\right)^{\frac{q-1}{q}}
\end{aligned}
$$

from which the inequality $\left\|Z_{1}+Z_{2}\right\|_{q} \geq\left\|Z_{1}\right\|_{q}+\left\|Z_{2}\right\|_{q}$ follows.

Remark 11. The functional $\|\cdot\|_{p^{\prime}}$ is not convex for $p^{\prime}<1$, hence one might assume that the set

$$
\left\{Z \in L^{1}: Z \geq 0, \mathbb{E} Z=1, H_{p^{\prime}}(Z) \leq \log \frac{1}{1-\alpha}\right\}
$$

of feasible densities in (11) is not convex for $p^{\prime}<1$ and $\alpha>0$. However, $H_{p^{\prime}}(Z) \leq \log \frac{1}{1-\alpha}$ rewrites as $\|Z\|_{p^{\prime}} \geq(1-\alpha)^{-\frac{1}{p^{\prime}}}$ for $p^{\prime}<1$. Thus the reverse Minkowski inequality guarantees that the set of feasible densities (15) is convex even for $p^{\prime}<1$.

Theorem 12. The domain of the risk measure $\mathrm{EV} @ \mathrm{R}_{\alpha}^{p}(\cdot)$ for $p<1$ is $L^{\infty}$, i.e., $\mathrm{EV} @ \mathrm{R}_{\alpha}^{p}(|Y|)<\infty$ if and only if $Y$ is bounded. Furthermore, the entropic risk measure $\mathrm{EV} @ \mathrm{R}_{\alpha}^{p}$ collapses to the essential supremum for $0<p<1$.

Proof. For $p \in(0,1)$ the conjugate Hölder exponent $p^{\prime}$ is negative and we may thus assume that $Z>0$. By Remark 5 we conclude that $H_{p^{\prime}}(Z) \leq 0$. The constraint $H_{p^{\prime}}(Z) \leq \log \frac{1}{1-\alpha}$ thus is trivial, as $\log \frac{1}{1-\alpha} \geq 0$ and it follows that the entropic risk measure $\mathrm{EV} @ \mathrm{R}_{\alpha}^{p}$ reduces to

$$
\mathrm{EV} @ \mathrm{R}_{\alpha}^{p}(Y)=\sup \{\mathbb{E} Y Z: Z \geq 0, \mathbb{E} Z=1\}=\operatorname{ess} \sup Y
$$

in this case.

Let us now consider the case $p<0$. Then its Hölder conjugate exponent satisfies $0<p^{\prime}<1$ and the constraint $H_{p^{\prime}}(Z) \leq \log \frac{1}{1-\alpha}$ is equivalent to $\mathbb{E} Z^{p^{\prime}} \geq\left(\frac{1}{1-\alpha}\right)^{p^{\prime}-1}=(1-\alpha)^{1-p^{\prime}}$. We may choose $\kappa>1$ large enough so that

$$
\left(1-\frac{1}{\kappa}\right)^{p^{\prime}}>(1-\alpha)^{1-p^{\prime}}
$$


For $\beta \in(0,1)$ consider random variables $Z_{\beta}$ with

$$
P\left(Z_{\beta}=\frac{1}{\kappa \beta}\right)=\beta \text { and } P\left(Z_{\beta}=\frac{1-\frac{1}{\kappa}}{1-\beta}\right)=1-\beta
$$

The random variable $Z_{\beta}$ is a density, as $Z_{\beta}>0$ and $\mathbb{E} Z_{\beta}=1$. For the random variables $Z_{\beta}$ it thus holds that

$$
\mathbb{E} Z_{\beta}^{p^{\prime}}=\beta\left(\frac{1}{\kappa \beta}\right)^{p^{\prime}}+(1-\beta)\left(\frac{1-\frac{1}{\kappa}}{1-\beta}\right)^{p^{\prime}} \underset{\beta \rightarrow 0}{\longrightarrow}\left(1-\frac{1}{\kappa}\right)^{p^{\prime}}>(1-\alpha)^{1-p^{\prime}}
$$

We thus may choose $\hat{\beta}<1$ so that for $\beta<\hat{\beta}$ we have that $\mathbb{E} Z_{\beta}^{p^{\prime}}>(1-\alpha)^{1-p^{\prime}}$.

Finally let $Y$ be an unbounded random variable. Without loss of generality we may assume that $Y \geq 0$. Then, for each $n \in \mathbb{N}$, the set $B_{n}:=\{Y \geq n\}$ has strictly positive probability and we set $\beta_{n}:=P\left(B_{n}\right)>0$. The variable

$$
Z_{\beta_{n}}(\omega):= \begin{cases}\frac{1}{\kappa \beta_{n}} & \text { if } \omega \in B_{n} \\ \frac{1-\frac{1}{\kappa}}{1-\beta_{n}} & \text { if } \omega \notin B_{n}\end{cases}
$$

is feasible and it holds that $\mathbb{E} Y Z_{\beta_{n}} \geq \frac{n}{\kappa}$ and thus $\operatorname{EV} @ \mathrm{R}_{\alpha}^{p}(Y) \geq \frac{n}{\kappa}$. This proves that $Y \notin L^{\infty}$ implies $\mathrm{EV} @ \mathrm{R}_{\alpha}^{p}(Y)=\infty$. The converse implication follows directly from Hölder's inequality.

It is now clear that for $p>1$ the risk measures EV@ $\mathrm{R}_{\alpha}^{p}$ have the domain $L^{p}$ and for these spaces the dual spaces are known, they are $L^{p^{\prime}}$ spaces, where $p^{\prime}$ is the Hölder conjugate of $p$. For $p=\infty$ we have the special case that EV@R can be defined on a space larger than $L^{\infty}$, this is studied in Ahmadi-Javid and Pichler (2017).

In what follows we address the duality relations of the Entropic Value-at-Risk for $p<1$.

\section{Dual representation of entropic risk measures}

In this section we develop representations of entropic risk measures which are dual to the expression given in Definition 8. This characterization allows us to deduce continuity properties of EV@R as well as to compare the entropic risk measures with Hölder norms. In what follows we discuss the three cases $p \geq 1, p \in(0,1)$ and $p<0$ separately.

\subsection{Infimum representation for $p \geq 1$}

The following theorem is originally due to Dentcheva et al. (2010). We state the result as it is similar to duality representations given below. We further use it to construct an explicit characterization of the dual norm and its corresponding Hahn-Banach functionals in Section 6 below.

Theorem 13 (Infimum representation for $p \geq 1$, cf. Dentcheva et al. (2010)). Let $\alpha \in(0,1)$, then the Entropic Value-at-Risk based on Rényi entropy for $p \in[1, \infty)$ has the representation

$$
\mathrm{EV} @ \mathrm{R}_{\alpha}^{p}(Y)=\inf _{t \in \mathbb{R}}\left\{t+\left(\frac{1}{1-\alpha}\right)^{1 / p} \cdot\left\|(Y-t)_{+}\right\|_{p}\right\}
$$


Remark 14. Note that the previous setting includes the case $p=1$ as a special case and we have the identity

$$
\mathrm{AV} @ \mathrm{R}_{\alpha}(Y)=\inf _{t \in \mathbb{R}}\left\{t+\frac{1}{1-\alpha} \cdot \mathbb{E}(Y-t)_{+}\right\}
$$

given in Ogryczak and Ruszczyński (2002). The result (16) deduces as well from

$$
\mathrm{EV} @ \mathrm{R}_{\alpha}^{p}(Y)=\sup \left\{\mathbb{E} Y Z: Z \geq 0, \mathbb{E} Z=1 \text { and }\|Z\|_{p^{\prime}} \leq\left(\frac{1}{1-\alpha}\right)^{\frac{p^{\prime}-1}{p^{\prime}}}\right\}
$$

and Pichler (2017, Theorem 3.1).

Dentcheva et al. (2010) also relate the optimal density $Z^{*}$ of (11) and the optimizer $t^{*}$ of (16) by

$$
Z^{*}=\frac{\left(Y-t^{*}\right)_{+}^{p-1}}{\mathbb{E}\left(Y-t^{*}\right)_{+}^{p-1}}
$$

if $t^{*}<\operatorname{ess} \sup Y$. For $t^{*}=\operatorname{ess} \sup Y$ they give the optimal density $Z^{*}=\frac{1}{p_{\max }} \mathbb{1}_{\{Y=\operatorname{ess} \sup Y\}}$ with $p_{\max }:=$ $P(Y=\operatorname{ess} \sup Y)$.

\subsection{Infimum representation for $p<0$}

To elaborate the dual representation in analogy to (16) for $p<0$ we discuss the function $f:(\operatorname{ess} \sup Y, \infty) \rightarrow \mathbb{R}$ given by

$$
f(t)=t-\left(\frac{1}{1-\alpha}\right)^{1 / p} \cdot\|t-Y\|_{p}
$$

where $p<0$ and $Y \in L^{\infty}$. The function $f(\cdot)$ has the following property.

Proposition 15. The following are equivalent for $p<0$ and $Y \in L^{\infty}$ :

(i) The infimum in (19) is attained at some $t^{*}>\operatorname{ess} \sup Y$,

(ii) $P(Y=\operatorname{ess} \sup Y)<1-\alpha$.

Furthermore, if $t^{*}=\operatorname{ess} \sup Y$, then it holds that $\inf _{t>\operatorname{ess} \sup Y} f(t)=\operatorname{ess} \sup Y$.

Proof. For $p<0$, the function $\|\cdot\|_{p}$ is concave by the reverse Minkowski inequality. The function (19) thus is convex and hence almost everywhere differentiable. For $t \in \mathbb{R}$ large enough the objective is monotone increasing, as we have

$$
\begin{aligned}
f(t) & =t-\left(\frac{1}{1-\alpha}\right)^{\frac{1}{p}}\left(\mathbb{E}(t-Y)^{p}\right)^{\frac{1}{p}}=t-\left(\frac{1}{1-\alpha}\right)^{\frac{1}{p}} \cdot t \cdot\left(\mathbb{E} 1-\frac{p Y}{t}\right)^{\frac{1}{p}}+O(1 / t) \\
& =t-\left(\frac{1}{1-\alpha}\right)^{\frac{1}{p}}(t-\mathbb{E} Y)+O(1 / t)
\end{aligned}
$$

by successive Taylor series expansions; the infimum is hence attained at some $t^{*}<\infty$, as $\left(\frac{1}{1-\alpha}\right)^{\frac{1}{p}}<1$. 
The derivative $f^{\prime}(t)=1-(1-\alpha)^{-1 / p}\left(\mathbb{E}(t-Y)^{p}\right)^{\frac{1}{p}-1} \cdot \mathbb{E}(t-Y)^{p-1}$ is negative if and only if $(1-\alpha)^{1 / p}<$ $\left(\mathbb{E}(t-Y)^{p}\right)^{\frac{1-p}{p}} \cdot \mathbb{E}(t-Y)^{p-1}$. Now note that $1-p>0$ so that we get $(1-\alpha)^{\frac{1}{p(1-p)}} \cdot\|t-Y\|_{p-1}<\|t-Y\|_{p}$. Define $A_{\varepsilon}:=\{Y+\varepsilon \geq \operatorname{ess} \sup Y\}$ and set $Y_{\max }:=\operatorname{ess} \sup Y$. Then we have for large $t$ that

$$
\left(t-Y_{\max }\right)^{p-1} P\left(A_{\varepsilon}\right)+\varepsilon^{p-1} P\left(A_{\varepsilon}^{\mathrm{c}}\right) \geq \mathbb{E}(t-Y)^{p-1}
$$

whenever $t>Y_{\max }$. This implies

$$
\begin{aligned}
(1-\alpha)^{\frac{1}{p(1-p)}} \cdot\left(\left(t-Y_{\max }\right)^{p-1} P\left(A_{\varepsilon}\right)+\varepsilon^{p-1} P\left(A_{\varepsilon}^{\mathrm{c}}\right)\right)^{\frac{1}{p-1}} & \\
\leq & (1-\alpha)^{\frac{1}{p(1-p)}} \cdot\|t-Y\|_{p-1}<\|t-Y\|_{p} \leq\left(\left(t-Y_{\max }\right)^{p} P\left(A_{\varepsilon}\right)\right)^{\frac{1}{p}} .
\end{aligned}
$$

Dividing the quantity by $t-Y_{\max }$ we obtain further

$$
(1-\alpha)^{\frac{1}{p(1-p)}} \cdot\left(P\left(A_{\varepsilon}\right)+\varepsilon^{p-1} P\left(A_{\varepsilon}^{\mathrm{c}}\right)\left(t-Y_{\max }\right)^{1-p}\right)^{\frac{1}{p-1}}<P\left(A_{\varepsilon}\right)^{\frac{1}{p}} .
$$

Letting $t \searrow Y_{\max }$ yields $(1-\alpha)^{\frac{1}{p(1-p)}} \cdot P\left(A_{\varepsilon}\right)^{\frac{1}{p-1}}<P\left(A_{\varepsilon}\right)^{\frac{1}{p}}$, which is equivalent to $(1-\alpha)>P\left(A_{\varepsilon}\right)$ and letting $\varepsilon \searrow 0$ give $1-\alpha>P\left(A_{0}\right)=P(Y=\operatorname{ess} \sup Y)$. Note that strict inequality holds, since $P\left(A_{\varepsilon}\right) \geq P\left(A_{0}\right)$ for all $\varepsilon>0$. Therefore $t^{*}>\operatorname{ess} \sup Y$ implies that $1-\alpha>P(Y=\operatorname{ess} \sup Y)$.

The converse implication $(i i) \Longrightarrow(i)$ is proven similarly.

To see the remaining statement set $A:=\{Y=\operatorname{ess} \sup Y\}$. By the previous results we know that the objective function $f$ is increasing on its domain. Therefore the infimum of (19) is a limit and

$$
\inf _{t>\text { ess sup } Y}\left\{t-\left(\frac{1}{1-\alpha}\right)^{1 / p} \cdot\|t-Y\|_{p}\right\}=\lim _{t \backslash \text { ess sup } Y} t-\lim _{t \searrow \text { ess sup } Y}\left(\frac{1}{1-\alpha}\right)^{1 / p} \cdot\|t-Y\|_{p} .
$$

But for the second limit we have

$$
\begin{aligned}
0 \leq \lim _{t \backslash \text { ess sup } Y}\|t-Y\|_{p} & \leq \lim _{t \backslash \text { ess sup } Y}\left((t-\operatorname{ess} \sup Y)^{p} P(A)\right)^{\frac{1}{p}} \\
& =\lim _{t \searrow \text { ess sup } Y}(t-\operatorname{ess} \sup Y) P(A)^{\frac{1}{p}}=0,
\end{aligned}
$$

which proves that $\inf _{t>\operatorname{ess} \sup Y} f(t)=\operatorname{ess} \sup Y$ and concludes the proof.

Using the characterization of the optimal value of $f(\cdot)$ in (19) we are now ready to prove the infimum representation of $\mathrm{EV} @ \mathrm{R}_{\alpha}^{p}$ for $p<0$.

Theorem 16 (Infimum representation for $p<0)$. Let $\alpha \in(0,1)$, then the Entropic Value-at-Risk based on Rényi entropy $\left(\mathrm{EV} @ \mathrm{R}_{\alpha}^{p}\right)$ for $p<0$ has the representation

$$
\mathrm{EV} @ \mathrm{R}_{\alpha}^{p}(Y)=\inf _{t>\operatorname{ess} \sup Y}\left\{t-\left(\frac{1}{1-\alpha}\right)^{1 / p} \cdot\|t-Y\|_{p}\right\} .
$$

Proof. Let $f(\cdot)$ denote the objective function in (20). From the previous proposition it is clear that the minimizer $t^{*}$ of $f(\cdot)$ either satisfies $t^{*}=\operatorname{ess} \sup Y$ or $t^{*}>\operatorname{ess} \sup Y$. First assume that $t^{*}>\operatorname{ess} \sup Y$. The random variable $Z:=\frac{1}{c}\left(t^{*}-Y\right)^{p-1}$ with $c:=\mathbb{E}\left(t^{*}-Y\right)^{p-1}$ has expectation 1 and $Z>0$, i.e., $Z$ is a density. 
By definition of $Z$, Hölder's inequality is an equality for $Z$ and $\left(t^{*}-Y\right)$, i.e., $\mathbb{E}\left(t^{*}-Y\right) Z=\left\|t^{*}-Y\right\|_{p}\|Z\|_{p^{\prime}}$, as

$$
|Z|^{p^{\prime}} \mathbb{E}\left(t^{*}-Y\right)^{p}=\left|t^{*}-Y\right|^{p} \mathbb{E} Z^{p^{\prime}} .
$$

Furthermore, for the optimizer $t^{*}$ of the objective function $f(\cdot)$ it holds that $f^{\prime}\left(t^{*}\right)=0$, which is

$$
(1-\alpha)^{\frac{1}{p}}=\mathbb{E}\left(t^{*}-Y\right)^{p-1}\left(\mathbb{E}\left(t^{*}-Y\right)^{p}\right)^{-\frac{1}{p^{\prime}}} .
$$

Therefore,

$$
\begin{aligned}
\mathbb{E} Y Z & =t^{*}-\mathbb{E}\left(t^{*}-Y\right) Z=t^{*}-\left\|t^{*}-Y\right\|_{p}\|Z\|_{p^{\prime}}=t^{*}-\left(\frac{1}{1-\alpha}\right)^{\frac{1}{p}}\left\|t^{*}-Y\right\|_{p} \\
& =\inf _{t>\operatorname{ess} \sup Y}\left\{t-\left(\frac{1}{1-\alpha}\right)^{1 / p} \cdot\|t-Y\|_{p}\right\}
\end{aligned}
$$

establishing that EV@ $\mathrm{R}_{\alpha}^{p}(Y) \geq \inf _{t>\operatorname{ess} \sup Y}\left\{t-\left(\frac{1}{1-\alpha}\right)^{1 / p} \cdot\|t-Y\|_{p}\right\}$. Suppose for a moment that $Z$ is not a maximizing density in (11), then there is another density $\widehat{Z}$ satisfying the moment constraints which maximizes (11), but

$$
\mathbb{E} Y \widehat{Z}=t^{*}-\mathbb{E}\left(t^{*}-Y\right) \widehat{Z}<t^{*}-\left\|t^{*}-Y\right\|_{p}\|\widehat{Z}\|_{p^{\prime}}
$$

Since $H_{p^{\prime}}(Z) \leq \log \left(\frac{1}{1-\alpha}\right)$ is equivalent to $\|Z\|_{p^{\prime}} \geq\left(\frac{1}{1-\alpha}\right)^{\frac{1}{p}}$, it follows that

$$
t^{*}-\left\|t^{*}-Y\right\|_{p}\|\widehat{Z}\|_{p^{\prime}}<t^{*}-\left(\frac{1}{1-\alpha}\right)^{\frac{1}{p}}\left\|t^{*}-Y\right\|_{p}=\mathbb{E} Y Z
$$

contradicting the assumption that $\widehat{Z}$ maximizes (11) and thus EV@ $\mathrm{R}_{\alpha}^{p}(Y)=\mathbb{E} Y Z$.

Now assume that $t^{*}=\operatorname{ess} \sup Y$. By the previous proposition it follows that $P(Y=\operatorname{ess} \sup Y) \geq 1-\alpha$ and therefore define

$$
Z:=P(Y=\operatorname{ess} \sup Y)^{-1} \mathbb{1}_{\{Y=\operatorname{ess} \sup Y\}}
$$

Then $\mathbb{E} Z=1,\|Z\|_{p^{\prime}}=P(Y=\operatorname{ess} \sup Y)^{\frac{1-p^{\prime}}{p^{\prime}}}>(1-\alpha)^{\frac{1-p^{\prime}}{p^{\prime}}}$ and $\mathbb{E} Y Z=$ ess $\sup Y$. By the previous proposition we have $\operatorname{EV} @ \mathrm{R}_{\alpha}^{p}(Y) \geq \inf _{x>\operatorname{ess} \sup Y}\left\{t-\left(\frac{1}{1-\alpha}\right)^{1 / p} \cdot\|t-Y\|_{p}\right\}=\operatorname{ess} \sup Y$. Now consider any density $\widehat{Z}$ satisfying the moment constraints and let $t^{*}=\operatorname{ess} \sup Y$. Then

$$
\mathbb{E} Y \widehat{Z}=t^{*}-\mathbb{E}\left(t^{*}-Y\right) \widehat{Z} \leq t^{*}=\operatorname{ess} \sup Y=\inf _{x>\operatorname{ess} \sup Y}\left\{t-\left(\frac{1}{1-\alpha}\right)^{1 / p} \cdot\|t-Y\|_{p}\right\}
$$

where the last equation follows by the assumption $t^{*}=$ ess $\sup Y$ and Proposition 15. This establishes the infimum representation. 


\subsection{Infimum representation for $p \in(0,1)$}

From Theorem 12 we know that for $0<p<1$ the entropic risk measure EV@ $\mathrm{R}_{\alpha}^{p}$ does not depend on $\alpha$ or $p$. The corresponding infimum representation is

$$
\mathrm{EV} @ \mathrm{R}_{\alpha}^{p}(Y)=\|Y\|_{\infty}=\inf _{t \geq \operatorname{ess} \sup Y}\left\{t+\left\|(Y-t)_{+}\right\|_{p}\right\}
$$

as the infimum is always attained for $t=\operatorname{ess} \sup Y$. As this case is trivial we will not consider it throughout the remainder of this paper.

\section{Monotonicity and EV@R-norms}

Based on the properties of the Rényi entropy and the infimum representation of EV@R we deduce limiting risk measures of EV@ $\mathrm{R}^{p}$ as well as a general monotonicity in the dual parameter $p^{\prime}$. We further show that the Entropic Value-at-Risk based on Rényi entropy is convex in its dual order $p^{\prime}$.

Lemma 17 (The special case $p=1$ ). For $\alpha \in(0,1)$ and $Y \in L^{1}$ we have

$$
\mathrm{EV} @ \mathrm{R}_{\alpha}^{p}(Y) \underset{p \downarrow 1}{\longrightarrow} \mathrm{AV} @ \mathrm{R}_{\alpha}(Y)
$$

Proof. From the definition of Rényi entropy we have

$$
\lim _{p^{\prime} \rightarrow \infty} H_{p^{\prime}}(Z)=\lim _{p^{\prime} \rightarrow \infty} \frac{p^{\prime}}{p^{\prime}-1} \log \|Z\|_{p^{\prime}}=\log \|Z\|_{\infty}
$$

so that the inequality $\log \|Z\|_{\infty}=\lim _{p^{\prime} \rightarrow \infty} H_{p^{\prime}}(Z) \leq \log \frac{1}{1-\alpha}$ is satisfied for every $Z$ satisfying the constraint in (11) and $p>1$. Therefore $Z \leq \frac{1}{1-\alpha}$, and consequently

$$
\lim _{p \rightarrow 1} \mathrm{EV} @ \mathrm{R}_{\alpha}^{p}(Y) \leq \operatorname{AV} @ \mathrm{R}_{\alpha}(Y)
$$

Consider further the generalized indicator function

$$
\mathbb{1}_{\left\{Y \geq \mathrm{V} @ \mathrm{R}_{\alpha}(Y)\right\}}^{\alpha}:= \begin{cases}\mathbb{1}_{\left\{Y \geq \mathrm{V} @ \mathrm{R}_{\alpha}(Y)\right\}} & \text { if } P\left(Y=\mathrm{V} @ \mathrm{R}_{\alpha}(Y)\right)=0 \\ \mathbb{1}_{\left\{Y>\mathrm{V} @ \mathrm{R}_{\alpha}(Y)\right\}}+\frac{P\left(Y \leq \mathrm{V} @ \mathrm{R}_{\alpha}(Y)\right)-\alpha}{P\left(Y=\mathrm{V} @ \mathrm{R}_{\alpha}(Y)\right)} \mathbb{1}_{\left\{Y=\mathrm{V} @ \mathrm{R}_{\alpha}(Y)\right\}} & \text { if } P\left(Y=\mathrm{V} @ \mathrm{R}_{\alpha}(Y)\right)>0\end{cases}
$$

and define the random variable $Z=\frac{1}{1-\alpha} \mathbb{1}_{\left\{Y \geq \mathrm{V} @ \mathrm{R}_{\alpha}(Y)\right\}}^{\alpha}$. Then $Z$ is a density with $H_{\infty}(Z)=\log \frac{1}{1-\alpha}$ and thus $\lim _{p \rightarrow 1} \mathrm{EV} @ \mathrm{R}_{\alpha}^{p}(Y) \geq \mathrm{AV} @ \mathrm{R}_{\alpha}(Y)$. Hence the assertion.

Lemma 18 (Special case $p=0)$. For $\alpha \in(0,1)$ and $Y \in L^{\infty}$ it holds that

$$
\mathrm{EV} @ \mathrm{R}_{\alpha}^{p}(Y) \underset{p \uparrow 0}{\longrightarrow}\|Y\|_{\infty}
$$

Proof. Set $A_{\varepsilon}:=\left\{Y>\|Y\|_{\infty}-\varepsilon\right\}$ for $\varepsilon>0$. Set $\lambda:=\frac{1}{2} P\left(A_{\varepsilon}\right)$ and consider the density

$$
Z:=\frac{1}{2 \lambda} \mathbb{1}_{A_{\varepsilon}}
$$

Note now that $x^{p^{\prime}} \underset{p^{\prime} \rightarrow 0}{\longrightarrow} 1$ for every $x>0$, so it is possible to find $p^{\prime}>0$ small enough so that $\frac{1}{p^{\prime}-1} \log \mathbb{E} Z^{p^{\prime}} \leq$ $\log \frac{1}{1-\alpha}$. It holds that $\mathbb{E}|Y| Z \geq \frac{1}{2 \lambda}\left(\|Y\|_{\infty}-\varepsilon\right) P\left(A_{\varepsilon}\right)=\|Y\|_{\infty}-\varepsilon$, from which the assertion follows. 


\subsection{Monotonicity and Convexity}

The following theorem states that the Average Value-at-Risk and the Entropic Value-at-Risk are extremal cases for the risk measure based on Rényi entropy. It is more convenient to state the result in $p^{\prime}$ than $p$. This is due to monotonicity of $p^{\prime} \mapsto H_{p^{\prime}}(Z)$ from Lemma 4 and the fact that $p^{\prime}$ is less than 0 whenever $p \in(0,1)$.

Theorem 19 (Monotonicity). Let $\alpha \in(0,1)$, then for $p_{1}^{\prime}<0<p_{2}^{\prime}<1 \leq p_{3}^{\prime} \leq p_{4}^{\prime}$ and their corresponding Hölder conjugates $p_{i}=\frac{p_{i}^{\prime}}{p_{i}^{\prime}-1}$ it holds that

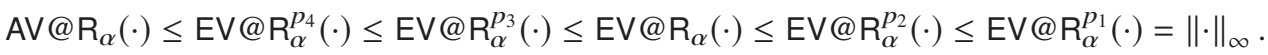

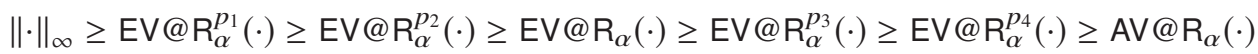

The mapping $p^{\prime} \mapsto \mathrm{EV} @ \mathrm{R}_{\alpha}^{p}(Y)$ is continuous for all $Y$, for which the expression is finite.

Proof. The result follows from Lemmas 17, 18 and 4. The last equality follows from Theorem 12. Continuity follows from Lemma 7.

From Theorem 19 we know that $p^{\prime} \mapsto \mathrm{EV} @ \mathrm{R}_{\alpha}^{p}$ is decreasing. We now show that this mapping is not only monotone but logarithmically convex.

Theorem 20 (Log-convexity of the Entropic Value-at-Risk). For $1<p_{0}, p_{1}$ and $0 \leq \lambda \leq 1$ define $p_{\lambda}^{\prime}:=$ $(1-\lambda) p_{0}^{\prime}+\lambda p_{1}^{\prime}$, where $p_{0}^{\prime}\left(p_{1}^{\prime}\right.$, resp. $)$ is the Hölder conjugate exponent of $p_{0}\left(p_{1}\right.$, resp. $)$. Then, for $Y \in L^{\infty}$, $\alpha \in(0,1)$ and $p_{\lambda}:=\frac{p_{\lambda}^{\prime}}{p_{\lambda}^{\prime}-1}$ we have that

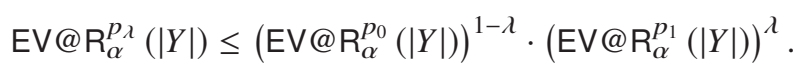

That is, the Entropic Value-at-Risk is logarithmically convex in its conjugate order.

Proof. The rather technical proof can be found in the appendix. There we first derive a compact representation of the derivative $\frac{\mathrm{d}}{\mathrm{d} p^{\prime}} \mathrm{EV} @ \mathrm{R}_{\alpha}^{\frac{p^{\prime}}{p^{\prime}-1}}(Y)$ and consequently show first convexity and then log-convexity.

Remark 21. We emphasize that the above result on logarithmic convexity of the function $p^{\prime} \mapsto \operatorname{EV} @ \mathrm{R}_{\alpha}^{\frac{p^{\prime}}{p^{\prime}-1}}(Y)$ does not extent to the case $p^{\prime} \in(0,1)$. For $p^{\prime}<0$, in contrast, we have $\operatorname{EV} @ \mathrm{R}_{\alpha}^{\frac{p^{\prime}}{p^{\prime}-1}}(Y)=\operatorname{ess} \sup (Y)$ and convexity hence is obvious.

\subsection{Comparison with Hölder norms}

The remainder of this section is concerned with the norms generated by EV@R. We show that the EV@ $\mathrm{R}_{\alpha^{-}}^{p}$ norm is equivalent to Hölder norms $(p \neq \infty)$, irrespective of the confidence level $\alpha$. The norms are equivalent for varying confidence level $\alpha$ and $p$ fixed.

Theorem 22 (Comparison with $\left.L^{p}\right)$. The space $\left(L^{p}, \mathrm{EV} @ \mathrm{R}_{\alpha}^{p}(|\cdot|)\right)$ is a Banach space for each $\alpha \in(0,1)$ and

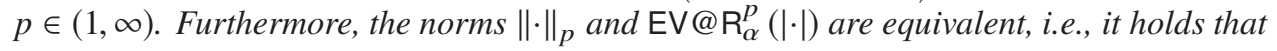

$$
C\|\cdot\|_{p} \leq \operatorname{EV} @ \mathrm{R}_{\alpha}^{p}(|\cdot|) \leq\left(\frac{1}{1-\alpha}\right)^{\frac{1}{p}}\|\cdot\|_{p},
$$

where $C=1 \wedge\left(\left(\frac{1}{1-\alpha}\right)^{\frac{1}{p-1}}-1\right)^{\frac{p-1}{p}}$. The inequality (22) is sharp. 
Proof. The right inequality follows directly from Hölder's inequality, cf. (13). To see that the right inequality is sharp consider the random variable $Y=\mathbb{1}_{A}$ with $P(A)=1-\alpha$. Then it holds that $\mathrm{EV} @ \mathrm{R}_{\alpha}^{p}(Y)=1$ and $\|Y\|_{p}=(1-\alpha)^{\frac{1}{p}}$.

For the left inequality consider first the case where the optimal $t^{*} \geq 0$ so that

$$
\mathrm{EV} @ \mathrm{R}_{\alpha}^{p}(Y) \geq \inf _{t>0} t+\left\|(Y-t)_{+}\right\|_{p}=\|Y\|_{p} .
$$

The case $t^{*}<0$ is more complicated. Consider a simple random variable $Y=\sum_{i} \lambda_{i} \mathbb{1}_{A_{i}}$, then

$$
C\|Y\|_{p}=C\left\|\sum_{i} \lambda_{i} \mathbb{1}_{A_{i}}\right\|_{p} \leq C \sum_{i} \lambda_{i}\left\|\mathbb{1}_{A_{i}}\right\|_{p} \leq \sum_{i} \lambda_{i} \operatorname{EV} @ \mathrm{R}_{\alpha}^{p}\left(\mathbb{1}_{A_{i}}\right)=\operatorname{EV} @ \mathrm{R}(Y),
$$

therefore it is enough to consider random variables of the form $Y=C \mathbb{1}_{A}$. Without loss of generality we may assume that $\|Y\|_{p}=1$ and consider $Y_{A}=\left(\frac{1}{\varepsilon}\right)^{\frac{1}{p}} \mathbb{1}_{A}$ where $\varepsilon:=P(A)$. Define the quantity $t^{*}=-\left((1-\alpha)^{\frac{1}{1-p}}-1\right)^{-\frac{1}{p}}$, which is negative for $\alpha<1-2^{1-p}$. Hence, for $\alpha<1-2^{1-p}$, we have that

$$
\mathrm{EV} @ \mathrm{R}_{\alpha}^{p}\left(Y_{\varepsilon}\right) \leq t^{*}+\left(\frac{1}{1-\alpha}\right)^{\frac{1}{p}}\left((1-\varepsilon)\left(-t^{*}\right)^{p}+\varepsilon\left(\left(\frac{1}{\varepsilon}\right)^{\frac{1}{p}}-t^{*}\right)^{p}\right)^{\frac{1}{p}} .
$$

The right hand side is differentiable in $\varepsilon$ with derivative

$$
\begin{aligned}
\frac{\mathrm{d}}{\mathrm{d} \varepsilon} f(\varepsilon)= & \left(\frac{1}{1-\alpha}\right)^{\frac{1}{p}} \frac{1}{p}\left[(1-\varepsilon)\left(-t^{*}\right)^{p}+\varepsilon\left((\varepsilon)^{-\frac{1}{p}}-t^{*}\right)\right]^{\frac{1}{p}-1} \\
& \times\left[-\left(-t^{*}\right)^{p}+\left(\left(\frac{1}{\varepsilon}\right)^{\frac{1}{p}}-t^{*}\right)^{p}+\varepsilon p\left(-\frac{1}{p} \varepsilon^{-\frac{1}{p}-1}\right)\left(\left(\frac{1}{\varepsilon}\right)^{\frac{1}{p}}-t^{*}\right)^{p-1}\right] .
\end{aligned}
$$

The first factor is positive, so that the derivative $\frac{\mathrm{d}}{\mathrm{d} \varepsilon} f(\varepsilon)$ is positive, if and only if

$$
\begin{aligned}
0 & \leq-\left(-t^{*}\right)^{p}+\left(\left(\frac{1}{\varepsilon}\right)^{\frac{1}{p}}-t^{*}\right)^{p}+\varepsilon p\left(-\frac{1}{p} \varepsilon^{-\frac{1}{p}-1}\right)\left(\left(\frac{1}{\varepsilon}\right)^{\frac{1}{p}}-t^{*}\right)^{p-1} \\
& =\left(\left(\frac{1}{\varepsilon}\right)^{\frac{1}{p}}-t^{*}\right)^{p-1}\left(-\varepsilon^{-\frac{1}{p}}+\varepsilon^{-\frac{1}{p}}-t^{*}\right)-\left(-t^{*}\right)^{p}
\end{aligned}
$$

However, this is the case, as it is a consequence of the binomial theorem. We now consider the limit

$$
\lim _{\varepsilon \rightarrow 0} t^{*}+\left(\frac{1}{1-\alpha}\right)^{\frac{1}{p}}\left((1-\varepsilon)\left(-t^{*}\right)^{p}+\varepsilon\left(\left(\frac{1}{\varepsilon}\right)^{\frac{1}{p}}-t^{*}\right)^{p}\right)^{\frac{1}{p}}=t^{*}+\left(\frac{1}{1-\alpha}\right)^{\frac{1}{p}}\left(\left(-t^{*}\right)^{p}+1\right)^{\frac{1}{p}},
$$

which is a lower bound for $\operatorname{EV} @ \mathrm{R}_{\alpha}^{p}\left(Y_{\varepsilon}\right)$. This is the optimal bound, since

$$
t^{*} \in \underset{t \in \mathbb{R}}{\arg \min } t+\left(\frac{1}{1-\alpha}\right)^{\frac{1}{p}}\left((-t)^{p}+1\right)^{\frac{1}{p}}=-\left((1-\alpha)^{\frac{1}{1-p}}-1\right)^{-\frac{1}{p}} .
$$

The optimal constant in (22) is thus given by $1 \wedge t^{*}+\left(\frac{1+\left(-t^{*}\right)^{p}}{1-\alpha}\right)^{\frac{1}{p}}$, i.e., $C=1 \wedge\left((1-\alpha)^{\frac{1}{1-p}}-1\right)^{\frac{p-1}{p}}$. 
Theorem 23 (Comparison with $\left.L^{\infty}\right)$. For $\alpha \in(0,1)$ and $p<1$, the norms $\mathrm{EV} @ \mathrm{R}_{\alpha}^{p}(|\cdot|)$ and $\|\cdot\|_{\infty}$ are equivalent. Indeed, we have that

$$
c\|\cdot\|_{\infty} \leq \mathrm{EV} @ \mathrm{R}_{\alpha}^{p}(|\cdot|) \leq\|\cdot\|_{\infty},
$$

where the constant $c=1-(1-\alpha)^{-\frac{1}{p}}$ is sharp.

Proof. By Theorem 12 it is sufficient to consider the case $p<0$. Then, by Theorem 19, EV@ $\mathrm{R}_{\alpha}^{p}(|Y|) \leq\|Y\|_{\infty}$ for all $Y \in L^{\infty}$.

Without loss of generality let $Y \geq 0$ and $\|Y\|_{\infty}=1$. Let $t^{*}$ be the minimizer from the infimum representation (26). By Proposition 15 we know that $t^{*} \geq\|Y\|_{\infty}=1$ and thus

$$
t^{*}=\left\|t^{*}\right\|_{p} \geq\left\|t^{*}-Y\right\|_{p} .
$$

It follows that

$$
\begin{aligned}
\mathrm{EV} @ \mathrm{R}_{\alpha}^{p}(|Y|) & =\inf _{t>\operatorname{ess} \sup (Y)}\left\{t-\left(\frac{1}{1-\alpha}\right)^{1 / p} \cdot\|t-Y\|_{p}\right\} \geq t^{*}-\left(\frac{1}{1-\alpha}\right)^{1 / p} t^{*} \\
& =t^{*}\left(1-(1-\alpha)^{-\frac{1}{p}}\right) \geq 1-(1-\alpha)^{-\frac{1}{p}} .
\end{aligned}
$$

We demonstrate that this constant is optimal for (24). Indeed, for every $\varepsilon>0$ consider the random variable $Y_{A}:=\mathbb{1}_{A}$ where $A$ is chosen such that $\varepsilon=P(A)$ for which

$$
\mathrm{EV} @ \mathrm{R}_{\alpha}^{p}\left(Y_{A}\right)=\inf _{t>1}\left\{t-\left(\frac{1}{1-\alpha}\right)^{\frac{1}{p}}\left((1-\varepsilon)(t)^{p}+\varepsilon(t-1)^{p}\right)^{\frac{1}{p}}\right\}
$$

Similar to the previous proof we see that the right hand side is increasing in $\varepsilon$ for each $t>1$ as

$$
\frac{\mathrm{d}}{\mathrm{d} \varepsilon}\left(t-\left(\frac{1}{1-\alpha}\right)^{\frac{1}{p}}\left((1-\varepsilon)(t)^{p}+\varepsilon(t-1)^{p}\right)^{\frac{1}{p}}\right)=-\left(\frac{1}{1-\alpha}\right)^{\frac{1}{p}}\left(-(t)^{p}+(t-1)^{p}\right)^{\frac{1}{p}}>0 .
$$

Fixing a $t>1$ we can evaluate the limit of the right hand side

$$
\lim _{\varepsilon \rightarrow 0}\left\{t-\left(\frac{1}{1-\alpha}\right)^{\frac{1}{p}}\left((1-\varepsilon)(t)^{p}+\varepsilon(t-1)^{p}\right)^{\frac{1}{p}}\right\}=t-\left(\frac{1}{1-\alpha}\right)^{\frac{1}{p}} t=t\left(1-(1-\alpha)^{-\frac{1}{p}}\right) .
$$

Taking the infimum over all feasible $t>1$ reveals that $c=1-(1-\alpha)^{-\frac{1}{p}}$ is the optimal constant in (24).

We investigate the EV@R for different confidence levels $\alpha$. It follows from (17) that EV@ $\mathrm{R}_{\alpha}^{p}(Y) \leq$ EV@ $\mathrm{R}_{\alpha^{\prime}}^{p}(Y)$ whenever $\alpha \leq \alpha^{\prime}$. In addition, the following holds true for nonnegative $Y \geq 0$.

Corollary 24 (Comparison for different risk levels). Let $\alpha \geq \alpha^{\prime}, p>1$ and $Y \geq 0$. Then it holds that

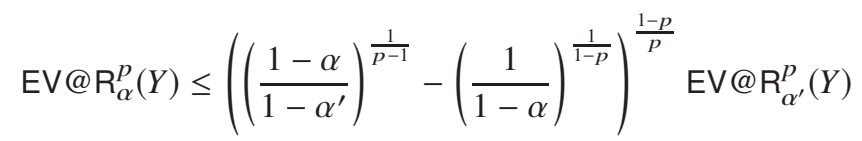

and for $p<0$,

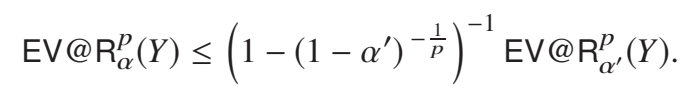

Proof. The proof is an application of Theorem 22 and Theorem 23, respectively. Therefore the EV@ $\mathrm{R}_{\alpha}^{p}$ norms are equivalent for different confidence levels. 


\section{Dual norms}

As mentioned in the introduction (cf. (4)) every coherent risk measure induces a semi-norm if applied to the absolute value of the argument. We have already seen that

$$
\|\cdot\|:=\mathrm{EV} @ \mathrm{R}_{\alpha}^{p}(|\cdot|)
$$

is in fact a norm on $L^{p}$ ( $L^{\infty}$ for $p<1$ ), respectively. For these new norms we consider the associated dual norm on the dual space $L^{p^{\prime}}$ given by

$$
\|Z\|_{\alpha, p^{\prime}}^{*}:=\sup _{\operatorname{EV} @ \mathrm{R}_{\alpha}^{p}(|Y|) \leq 1} \mathbb{E} Y Z .
$$

In what follows we give explicit representations for the dual norm $\|\cdot\|_{\alpha, p^{\prime}}^{*}$. Further, we describe the dual variables for which the maximum in (25) is attained (if available).

\subsection{Characterization of the dual norm}

The evaluation of the dual norm in (25) requires computing the supremum over an infinite dimensional space of random variables. Using the dual representations developed in Section 4 we can give an equivalent representation of those dual norms as a supremum over real numbers, thus facilitating the evaluation of those norms.

Corollary 25 (Corollary to Theorem 22). The norms $\|\cdot\|_{p^{\prime}}$ and $\|Z\|_{\alpha, p^{\prime}}^{*}$ are equivalent, it holds that

$$
(1-\alpha)^{\frac{p^{\prime}-1}{p^{\prime}}}\|Z\|_{p^{\prime}} \leq\|Z\|_{\alpha, p^{\prime}}^{*} \leq \frac{1}{C}\|Z\|_{p^{\prime}}
$$

where $C$ is the constant given in Theorem 22. The inequalities are sharp.

Proposition 26 (The explicit dual norm for $p>1$ ). Let $p>1$ and $p^{\prime}=\frac{p}{p-1}$. The dual norm $\|\cdot\|_{\alpha, p^{\prime}}^{*}$ of $\mathrm{EV} @ \mathrm{R}_{\alpha}^{p}(|\cdot|)$ is given by

$$
\|Z\|_{\alpha, p^{\prime}}^{*}=\sup _{t \in \mathbb{R}} \frac{\mathbb{E}\left(t+|Z|^{p^{\prime}-1}\right)_{+}|Z|}{t+\left(\frac{1}{1-\alpha}\right)^{\frac{1}{p}}\left\|\left(t+|Z|^{q-1}\right)_{+}-t\right\|_{p}} .
$$

Proof. We may assume that $Z \geq 0$ so that we may restrict (25) to $Y \geq 0$. Observe first that $\lambda \geq\|Z\|_{\alpha, p^{\prime}}^{*}$ is equivalent to $0 \geq \mathbb{E} Y Z-\lambda \mathrm{EV} @ \mathrm{R}_{\alpha}^{p}(|Y|)$ for all $Y \geq 0$. We maximize this expression with respect to $Y$. The Lagrangian of this maximization problem is

$$
L(Y, \lambda, \mu)=\mathbb{E} Y Z-\lambda \mathrm{EV} @ \mathrm{R}_{\alpha}^{p}(|Y|)-\mathbb{E} Y \mu,
$$

where $\mu$ is the Lagrange multiplier associated to the constraint $Y \geq 0$. The Lagrangian at the optimal $Y$ with optimizer $t^{*}$ for $\mathrm{EV} @ \mathrm{R}_{\alpha}^{p}(Y)$ is

$$
L(Y, \lambda, \mu)=\mathbb{E} Y Z-\lambda t^{*}-\lambda\left(\frac{1}{1-\alpha}\right)^{\frac{1}{P}}\left\|\left(Y-t^{*}\right)_{+}\right\|_{p}-\mathbb{E} Y \mu .
$$


The directional derivative of the Lagrangian in direction $H \in L^{p}$ at $Y$ is

$$
\frac{\partial}{\partial Y} L(Y, \lambda, \mu) H=\mathbb{E} H Z-\lambda\left(\frac{1}{1-\alpha}\right)^{\frac{1}{p}} \mathbb{E}\left[\left(Y-t^{*}\right)_{+}^{p}\right]^{\frac{1}{p}-1} \cdot \mathbb{E} H\left(Y-t^{*}\right)_{+}^{p-1}-\mathbb{E} H \mu .
$$

The derivative vanishes in every direction $H$ so that

$$
Z-\mu=c\left(Y-t^{*}\right)_{+}^{p-1}
$$

where $c=\left(\frac{1}{1-\alpha}\right)^{\frac{1}{p}} \mathbb{E}\left[\left(Y-t^{*}\right)_{+}^{p}\right]^{\frac{1}{p}-1}>0$. By complimentary slackness for the optimal $Y$ and $\mu$,

$$
Y>0 \Longleftrightarrow \mu=0 \Longleftrightarrow Z=c\left(Y-t^{*}\right)_{+}^{p-1}>c\left(-t^{*}\right)_{+}^{p-1},
$$

which is equivalent to $Y=\left(t^{*}+\left(\frac{Z}{c}\right)^{p^{\prime}-1}\right)_{+}$. Denote the optimal $Y$ in (25) by $Y_{\max }$ with optimizer $t^{*}$ of $\mathrm{EV} @ \mathrm{R}_{\alpha}^{p}\left(Y_{\max }\right)$. Then the above consideration implies that

$$
\begin{aligned}
\sup _{Y \neq 0} \frac{\mathbb{E} Y Z}{\operatorname{EV} @ \mathrm{R}_{\alpha}^{p}(|Y|)} & =\frac{\mathbb{E} Y_{\max } Z}{\operatorname{EV@~} \mathrm{R}_{\alpha}^{p}\left(\left|Y_{\max }\right|\right)} \\
& =\frac{\mathbb{E}\left(t^{*}+\left(\frac{Z}{c}\right)^{p^{\prime}-1}\right)_{+} Z}{t^{*}+\left(\frac{1}{1-\alpha}\right)^{\frac{1}{p}}\left\|\left(\left(t^{*}+\left(\frac{Z}{c}\right)^{p^{\prime}-1}\right)_{+}-t^{*}\right)_{+}\right\|_{p}} \\
& =\frac{\left(\frac{1}{c}\right)^{q-1} \mathbb{E}\left(c^{q-1} t^{*}+Z^{p^{\prime}-1}\right)_{+} Z}{t^{*}+\left(\frac{1}{c}\right)^{q-1}\left(\frac{1}{1-\alpha}\right)^{\frac{1}{p}}\left\|\left(\left(c^{q-1} t^{*}+Z^{q-1}\right)_{+}-c^{q-1} t^{*}\right)_{+}\right\|_{p}} .
\end{aligned}
$$

We assumed that $Z \geq 0$ and hence $\left(\left(t+Z^{p^{\prime}-1}\right)_{+}-t\right)_{+}=\left(Z^{p^{\prime}-1}+t\right)_{+}-t$. The dual norm then simplifies to

$$
\begin{aligned}
\sup _{Y \neq 0} \frac{\mathbb{E} Y Z}{\operatorname{EV} @ \mathrm{R}_{\alpha}^{p}(|Y|)} & =\frac{\mathbb{E}\left(c^{q-1} t^{*}+Z^{p^{\prime}-1}\right)_{+} Z}{c^{q-1} t^{*}+\left(\frac{1}{1-\alpha}\right)^{\frac{1}{p}}\left\|\left(c^{q-1} t^{*}+Z^{q-1}\right)_{+}-c^{q-1} t^{*}\right\|_{p}} \\
& =\sup _{t \in \mathbb{R}} \frac{\mathbb{E}\left(t+Z^{p^{\prime}-1}\right)_{+} Z}{t+\left(\frac{1}{1-\alpha}\right)^{\frac{1}{p}}\left\|\left(t+Z^{q-1}\right)_{+}-t\right\|_{p}}
\end{aligned}
$$

which concludes the proof.

In the case $p<0$ we deduce a similar result to Proposition 26. We can give the following characterization, which is again a supremum over one single parameter.

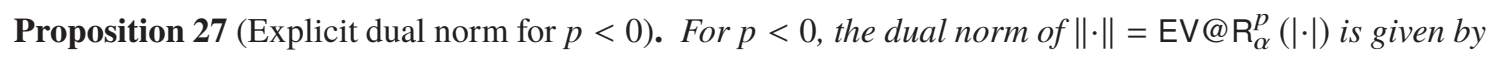

$$
\|Z\|_{\alpha, p^{\prime}}^{*}=\sup _{t>\operatorname{ess} \sup \left(Z^{p^{\prime}-1}\right)} \frac{\mathbb{E}\left(t-|Z|^{p^{\prime}-1}\right)|Z|}{t-\left(\frac{1}{1-\alpha}\right)^{\frac{1}{p}}\left\||Z|^{p^{\prime}-1}\right\|_{p} .}
$$


Proof. We may assume that $Z \geq 0$ so that we may restrict (25) to $Y \geq 0$. Observe first that that $\lambda \geq\|Z\|_{\alpha, p^{\prime}}^{*}$ is equivalent to $0 \geq \mathbb{E} Y Z-\lambda \mathrm{EV} @ \mathrm{R}_{\alpha}^{p}(|Y|)$ for all $Y \geq 0$. We maximize this expression with respect to $Y$. Then the Lagrange formulation of this maximization problem is

$$
\begin{aligned}
L(Y, \lambda, \mu) & =\mathbb{E} Y Z-\lambda \operatorname{EV} @ \mathrm{R}_{\alpha}^{p}(|Y|)-\mathbb{E} Y \mu \\
& =\mathbb{E} Y Z-\lambda t^{*}+\lambda\left(\frac{1}{1-\alpha}\right)^{\frac{1}{p}}\left\|t^{*}-Y\right\|_{p}-\mathbb{E} Y \mu,
\end{aligned}
$$

where $t^{*}$ is the optimizer of $\mathrm{EV} @ \mathrm{R}_{\alpha}^{p}(Y)$ and $\mu$ the Lagrange multiplier associated to the constraint $Y \geq 0 . L$ is differentiable in each direction and the directional derivative in $Y$ in the direction $H$ is

$$
\frac{\partial}{\partial Y} L(Y, \lambda, \mu) H=\mathbb{E} H Z-\lambda\left(\frac{1}{1-\alpha}\right)^{\frac{1}{p}} \mathbb{E}\left[\left(t^{*}-Y\right)^{p}\right]^{\frac{1}{p}-1} \mathbb{E} H\left(t^{*}-Y\right)^{p-1}-\mathbb{E} H \mu .
$$

The derivative vanishes in every direction $H$ and consequently we get

$$
Z-\mu=\frac{1}{c}\left(t^{*}-Y\right)^{p-1}
$$

Here, $\frac{1}{c}=\lambda\left(\frac{1}{1-\alpha}\right)^{\frac{1}{p}} \mathbb{E}\left[\left(t^{*}-Y\right)^{p}\right]^{\frac{1}{p}-1}$. By complimentary slackness for optimal $Y$ and $\mu$, it follows that

$$
Y>0 \Longleftrightarrow \mu=0 \Longleftrightarrow Z=\frac{1}{c}\left(t^{*}-Y\right)^{p-1} .
$$

This shows that it is enough to consider $Y$ of the form $Y=t-(c Z)^{p^{\prime}-1}$ in the definition of the dual norm. Denote now the optimal $Y$ in (25) by $Y_{\max }$. Then the above implies that for the optimizer $t^{*}$ of $\operatorname{EV} @ \mathrm{R}_{\alpha}^{p}\left(Y_{\max }\right)$ we have

$$
Y_{\max }=t^{*}-(c Z)^{p^{\prime}-1} .
$$

And therefore the expression (25) reduces to

$$
\begin{aligned}
\sup _{Y \neq 0} \frac{\mathbb{E} Y Z}{\operatorname{EV} @ \mathrm{R}_{\alpha}^{p}(|Y|)}= & \frac{\mathbb{E} Y_{\max } Z}{\operatorname{EV} @ \mathrm{R}_{\alpha}^{p}\left(\left|Y_{\max }\right|\right)}=\frac{\mathbb{E}\left(t^{*}-(c Z)^{p^{\prime}-1}\right) Z}{t^{*}-\left(\frac{1}{1-\alpha}\right)^{\frac{1}{p}}\left\|(c Z)^{p^{\prime}-1}\right\|_{p}} \\
& =\frac{\mathbb{E}\left[\left(\frac{t^{*}}{c p^{\prime}-1}-Z^{p^{\prime}-1}\right) Z\right]}{\frac{t^{*}}{c^{p^{\prime}-1}}-\left(\frac{1}{1-\alpha}\right)^{\frac{1}{p}}\left\|Z^{p^{\prime}-1}\right\|_{p}} .
\end{aligned}
$$

Notice that $Y>0$ implies that $\frac{t^{*}}{c^{p^{\prime}-1}}>\operatorname{ess} \sup \left(Z^{p^{\prime}-1}\right)$ and thus

$$
\frac{\mathbb{E}\left[\left(\frac{t^{*}}{c^{p^{\prime}-1}}-Z^{p^{\prime}-1}\right) Z\right]}{\frac{t^{*}}{c^{p^{\prime}-1}}-\left(\frac{1}{1-\alpha}\right)^{\frac{1}{p}}\left\|Z^{p^{\prime}-1}\right\|_{p}}=\sup _{t>Z^{p^{\prime}-1}} \frac{\mathbb{E}\left[\left(t-Z^{p^{\prime}-1}\right) Z\right]}{t-\left(\frac{1}{1-\alpha}\right)^{\frac{1}{p}}\left\|Z^{p^{\prime}-1}\right\|_{p}},
$$

which shows the assertion. 


\subsection{Hahn-Banach functionals}

We now describe the Hahn-Banach functionals corresponding to $Y \in L^{p}$ and $Z \in L^{p^{\prime}}$ explicitly. This means we identify the random variable $Z \in L^{p^{\prime}}$ which maximizes

$$
\operatorname{EV} @ \mathrm{R}(|Y|)=\sup _{Z \neq 0} \frac{\mathbb{E} Y Z}{\|Z\|_{\alpha, p^{\prime}}^{*}}
$$

and the random variable $Y \in L^{p}$ which maximizes

$$
\|Z\|_{\alpha, p^{\prime}}^{*}=\sup _{Y \neq 0} \frac{\mathbb{E} Y Z}{\operatorname{EV} @ \mathrm{R}_{\alpha}^{p}(|Y|)} .
$$

Proposition 28. For $p>1$ let $Y \in L^{p}$ and suppose that there is an optimizer $t^{*} \in \mathbb{R}$ of

$$
\inf _{t \in \mathbb{R}}\left\{t+\left(\frac{1}{1-\alpha}\right)^{1 / p} \cdot\left\|(|Y|-t)_{+}\right\|_{p}\right\} .
$$

Then $Z^{\prime}:=\operatorname{sign}(Y) \cdot\left(|Y|-t^{*}\right)_{+}^{p-1}$ maximizes the expression

$$
\operatorname{EV} @ \mathrm{R}_{\alpha}^{p}(|Y|)=\sup _{Z \neq 0} \frac{\mathbb{E} Y Z}{\|Z\|_{\alpha, p^{\prime}}^{*}} .
$$

Proof. Without loss of generality we assume $Y \geq 0$. Then, by the definition of the dual norm, the definition of $Z^{\prime}$ and Eq. (18) in Remark 14 we have $\mathbb{E} Z^{\prime} \cdot E V @ \mathrm{R}_{\alpha}^{p}(|Y|)=\mathbb{E} Y Z^{\prime}$. It is therefore enough to verify that $\mathbb{E} Z^{\prime}=\left\|Z^{\prime}\right\|_{\alpha, p^{\prime}}^{*}$. Since $\frac{Z^{\prime}}{\mathbb{E} Z^{\prime}}$ is a density we have

$$
\left\|Z^{\prime}\right\|_{\alpha, p^{\prime}}^{*}=\sup _{Y \neq 0} \frac{\mathbb{E} Y Z^{\prime}}{\operatorname{EV} @ R_{\alpha}^{p}(Y)}=\frac{\mathbb{E} Y^{*} Z^{\prime}}{\mathbb{E} Y^{*} Z^{*}}=\mathbb{E} Z^{\prime},
$$

where $Y^{*}$ is the maximizer of the above supremum and $Z^{*}$ the optimal density for $Y^{*}$.

We now address the converse question, which is: given $Z$, what is the random variable $Y$ to achieve equality in (25)?

Proposition 29. For $p^{\prime}>1$ let $Z \in L^{p^{\prime}}$ and suppose that there is an optimal $t^{*} \in \mathbb{R}$ in

$$
\sup _{t \in \mathbb{R}} \frac{\mathbb{E}\left(t+|Z|^{p^{\prime}-1}\right)_{+}|Z|}{t+\left(\frac{1}{1-\alpha}\right)^{\frac{1}{p}}\left\|\left(t+|Z|^{p^{\prime}-1}\right)_{+}-t\right\|_{p}} .
$$

Then $Y^{\prime}:=\operatorname{sign}(Z) \cdot\left(t^{*}+|Z|^{p^{\prime}-1}\right)_{+}$satisfies the equality

$$
\mathbb{E} Y^{\prime} Z=\mathrm{EV} @ \mathrm{R}_{\alpha}^{p}\left(Y^{\prime}\right) \cdot\|Z\|_{\alpha, p^{\prime}}^{*} .
$$

Proof. Without loss of generality, we may assume that $Z \geq 0$. By assumption

$$
\|Z\|_{\alpha, p^{\prime}}^{*}=\frac{\mathbb{E}\left(t^{*}+|Z|^{p^{\prime}-1}\right)_{+}|Z|}{t^{*}+\left(\frac{1}{1-\alpha}\right)^{\frac{1}{p}}\left\|\left(\left(t^{*}+|Z|^{p^{\prime}-1}\right)-t^{*}\right)\right\|_{p} .}
$$


Then

$$
\mathbb{E} Y^{\prime} Z \leq\|Z\|_{\alpha, p^{\prime}}^{*} \mathrm{EV} @ \mathrm{R}_{\alpha}^{p}\left(Y^{\prime}\right) \leq\|Z\|_{\alpha, p^{\prime}}^{*}\left(t^{*}+\left(\frac{1}{1-\alpha}\right)^{\frac{1}{p}}\left\|\left(Y^{\prime}-t^{*}\right)_{+}\right\|_{p}\right)=\mathbb{E} Y^{\prime} Z,
$$

hence equality holds and the assertion follows.

We now derive the corresponding Hahn-Banach functionals for the dual norm $\|Z\|_{\alpha, p}^{*}$ for $p<0$. The proofs are analogous to the case $p>1$ in Proposition 28 (Proposition 29, resp.) and therefore are omitted.

Proposition 30. Let $Y \in L^{\infty}$ and $p<0$. Further suppose that there is an optimal $t^{*} \in \mathbb{R}$ in

$$
\inf _{t>\operatorname{ess} \sup Y}\left\{t-\left(\frac{1}{1-\alpha}\right)^{1 / p} \cdot\|t-|Y|\|_{p}\right\} .
$$

Then $Z^{\prime}:=\operatorname{sign}(Y) \cdot\left(t^{*}-|Y|\right)^{p-1}$ maximizes the expression

$$
\mathrm{EV} @ \mathrm{R}_{\alpha}^{p}(|Y|)=\sup _{Z \neq 0} \frac{\mathbb{E} Y Z}{\|Z\|_{\alpha, p^{\prime}}^{*}}
$$

Proposition 31. Let $Z \neq 0$ and $p<0$ and suppose that there is an optimal $t^{*}<\infty$ in

$$
\sup _{t>\operatorname{ess} \sup Z^{p^{\prime}-1}} \frac{\mathbb{E}\left(t-|Z|^{p^{\prime}-1}\right)|Z|}{t-\left(\frac{1}{1-\alpha}\right)^{\frac{1}{p}}\left\||Z|^{p^{\prime}-1}\right\|_{p} .}
$$

Then $Y^{\prime}:=\operatorname{sign}(Z) \cdot\left(t^{*}-|Z|^{p^{\prime}-1}\right)$ satisfies the equality

$$
\|Z\|_{\alpha, p^{\prime}}^{*} \cdot \operatorname{EV} @ \mathrm{R}_{\alpha}^{p}\left(Y^{\prime}\right)=\mathbb{E} Y^{\prime} Z .
$$

With the maximizer of the Hahn-Banach functionals at hand, we can give an alternative supremum representation of the Entropic Value-at-Risk based on Rényi entropy.

Corollary 32 (Dual representation of EV@R). The Entropic Value-at-Risk has the alternative dual representation

$$
\mathrm{EV} @ \mathrm{R}_{\alpha}^{p}(Y)=\sup \left\{\mathbb{E} Y Z, Z \geq 0, \mathbb{E} Z=1,\|Z\|_{\alpha, p^{\prime}}^{*} \leq 1\right\}
$$

where either $p>1$ or $p<0$.

Proof. Suppose that $p>1$ first. Then, by Proposition 26, the inequality $\|Z\|_{\alpha, p^{\prime}}^{*} \leq 1$ is equivalent to

$$
\frac{\mathbb{E}\left(t+|Z|^{p^{\prime}-1}\right)_{+}|Z|}{t+\left(\frac{1}{1-\alpha}\right)^{\frac{1}{p}}\left\|\left(t+|Z|^{p^{\prime}-1}\right)_{+}-t\right\|_{p}} \leq 1
$$

for all $t \in \mathbb{R}$. Setting $t=0$ it follows that

$$
\mathbb{E} Z^{p^{\prime}} \leq\left(\frac{1}{1-\alpha}\right)^{\frac{1}{p}}\left(\mathbb{E} Z^{p^{\prime}}\right)^{\frac{1}{p}}
$$




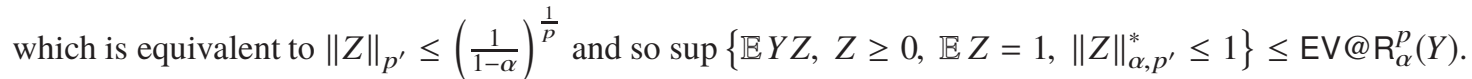

For $p<0$ the constraint $\|Z\|_{\alpha, p^{\prime}}^{*} \leq 1$ is equivalent to

$$
\frac{\mathbb{E}\left(t-|Z|^{p^{\prime}-1}\right)|Z|}{t-\left(\frac{1}{1-\alpha}\right)^{\frac{1}{p}}\left\||Z|^{p^{\prime}-1}\right\|_{p}}<1,
$$

which under the assumptions $Z \geq 0$ and $\mathbb{E} Z=1$ can be rewritten as $\mathbb{E} Z^{p^{\prime}} \geq\left(\frac{1}{1-\alpha}\right)^{\frac{1}{p}}\left\|Z^{p^{\prime}-1}\right\|_{p}$ or $\|Z\|_{p^{\prime}} \geq$ $\left(\frac{1}{1-\alpha}\right)^{\frac{p^{\prime}-1}{p^{\prime}}}$.

It remains to be shown that the maximizing $Z$ in (11) satisfies the constraint $\|Z\|_{\alpha, p^{\prime}}^{*} \leq 1$. In fact, by Theorem 13, we know that for $p>1$ the optimal $Z$ in $\operatorname{EV}_{0} \mathrm{R}_{\alpha}^{p}(Y)$ is given by $Z=\frac{\left(Y-t^{*}\right)_{+}^{p-1}}{\mathbb{E}\left(Y-t^{*}\right)_{+}^{p-1}}$, where $t^{*}$ is the optimizer of (16). By Proposition 29, the random variable $Y^{\prime}:=\operatorname{sign}(Z) \cdot\left(Z^{p^{\prime}-1}-t^{*}\right)$ satisfies

$$
\mathbb{E} Y^{\prime} Z=\mathbb{E V} @ \mathrm{R}_{\alpha}^{p}\left(Y^{\prime}\right)\|Z\|_{\alpha, p^{\prime}}^{*}
$$

and since $Z$ is feasible for (11) it follows that $\|Z\|_{\alpha, p^{\prime}}^{*}=\frac{\mathbb{E} Y^{\prime} Z}{\operatorname{EV} @ \mathrm{R}_{\alpha}^{p}\left(Y^{\prime}\right)} \leq 1$ by definition of $\mathrm{EV} @ \mathrm{R}_{\alpha}^{p}$. The same reasoning applies to the case $p<0$. Here, the optimal $Z$ is given by $Z=\frac{\left(t^{*}-Y\right)^{p-1}}{\mathbb{E}\left(t^{*}-Y\right)^{p-1}}$ according to Theorem 16 . We apply Proposition 31 to conclude the assertion.

\subsection{Kusuoka representation}

The $\mathrm{EV} @ \mathrm{R}_{\alpha}^{p}$ is a version independent coherent risk measure for which consequently a Kusuoka representation can be obtained (cf. Kusuoka (2001)). We derive the Kusuoka representation from its dual representation.

Proposition 33 (Kusuoka representation). The Kusuoka representation of the Entropic Value-at-Risk for $p>1$ or $p<0, \alpha \in[0,1)$ and $Y \in L^{p}\left(L^{\infty}\right)$, respectively is

$$
\mathrm{EV} @ \mathrm{R}_{\alpha}^{p}(Y)=\sup _{\mu} \int_{0}^{1} \operatorname{AV} @ \mathrm{R}_{x}(Y) \mu(\mathrm{d} x),
$$

where the supremum is among all probability measures $\mu$ on $[0,1)$ for which the function

$$
\sigma_{\mu}(u)=\int_{0}^{u} \frac{1}{1-v} \mu(\mathrm{d} v)
$$

satisfies

$$
\int_{0}^{1} \sigma_{\mu}(u)^{p^{\prime}} \mathrm{d} u \leq\left(\frac{1}{1-\alpha}\right)^{p^{\prime}-1}
$$

The supremum in (28) is attained for the measure $\mu_{\sigma *}$ associated with the distortion function

$$
\sigma^{*}(u):=F_{Z^{*}}^{-1}(u)=\mathrm{V} @ \mathrm{R}_{u}\left(Z^{*}\right),
$$

where $Z^{*}$ is the optimal random variable in $(11)$ and $F_{Z^{*}}^{-1}(u)=\mathrm{V} @ \mathrm{R}_{u}\left(Z^{*}\right)$ its generalized inverse. 
Proof. The representation follows from the supremum representation (11). Observe that the supremum is attained and for the maximizing density $Z^{*}$ in (11)

$$
\mathbb{E}\left(Z^{*}\right)^{p^{\prime}}=\left(\frac{1}{1-\alpha}\right)^{p^{\prime}-1}
$$

holds. By definition of $\sigma^{*}$ we have

$$
\mathbb{E}\left(Z^{*}\right)^{p^{\prime}}=\int_{0}^{1}\left(\sigma^{*}(u)\right)^{p^{\prime}} \mathrm{d} u .
$$

We define the measure $\mu^{*}(A):=\sigma^{*}(0) \cdot \delta_{0}(A)+\int_{A}(1-u) \mathrm{d} \sigma^{*}(u)$ for a measurable set $A \subseteq[0,1)$. For this measure we have

$$
\int_{0}^{u} \frac{1}{1-x} \mu^{*}(\mathrm{~d} x)=\sigma^{*}(0)+\int_{0}^{u} \frac{1}{1-v}(1-v) \mathrm{d} \sigma^{*}(v)=\sigma^{*}(u)
$$

and therefore $\sigma^{*}$ is feasible in the above supremum. Furthermore

$$
\begin{aligned}
\int_{0}^{1} \operatorname{AV} @ \mathrm{R}_{x}(Y) \mu^{*}(\mathrm{~d} x) & =\sigma^{*}(0) \mathrm{AV} @ \mathrm{R}_{0}(Y)+\int_{0}^{1} \frac{1}{1-x} \int_{x}^{1} F_{Y}^{-1}(u) \mathrm{d} u(1-x) \mathrm{d} \sigma^{*}(x) \\
& =\int_{0}^{1} \sigma^{*}(u) F_{Y}^{-1}(u) \mathrm{d} u \\
& =\mathbb{E} Y Z^{*}=\operatorname{EV} @ \mathrm{R}_{\alpha}^{p}(Y)
\end{aligned}
$$

and hence the assertion follows.

In this section we derive a computational convenient representation of the EV@R-dual norms and gave the explicit formulas for their maximizers. This allowed us to give another supremum representation for EV@R. We further elaborated on the Kusuoka representation of EV@R.

\section{Concluding Remarks}

This paper introduces entropic risk measures specified by a family of entropies. These risk measures are interesting in stochastic optimization and its applications as entropy allows the interpretation of information losses and the corresponding risk measures reflect ambiguity in terms of lost information.

Ahmadi-Javid (2012a) introduces the so-called Entropic Value-at-Risk as the tightest upper bound for the Value-at-Risk and the Average-Value-at-Risk using Chernoff's inequality. He expresses the classical Entropic Value-at-Risk by employing Shannon entropy.

We extend this work to the class of Rényi entropies and we show that the associated risk measures are monotone and continuous with respect to the Rényi order. The case studied in Ahmadi-Javid (2012a) arises as a special, limiting case. For the Rényi entropy of order larger than 1, the Rényi entropic risk measures interpolate the Average Value-at-Risk and the Entropic Value-at-Risk based on Shannon entropy. For the Rényi order smaller than 1 the Entropic Value-at-Risk based on Rényi entropy dominates the Entropic Value-at-Risk. The essential supremum is recovered as a limiting case as well.

Most importantly from a viewpoint of stochastic optimization we derive an equivalent infimum representation of the risk measures (11), where the infimum is considered over a real variable. This allows a efficient computation of stochastic programs employing these risk measures based on Rényi entropy. 
We further study the norms associated with entropic risk measures and elaborate the exact constants in comparing them with Hölder norms. In this way we relate them to higher order risk measures. We further explicit the formulas of the dual norms and the corresponding Hahn-Banach functionals. We use the duality results to derive alternative dual and Kusuoka representations and state the maximizing densities explicitly.

\section{Acknowledgment}

We wish to thank the editor and the referees of this journal for their time and commitment in assessing the paper.

\section{References}

A. Ahmadi-Javid. Entropic Value-at-Risk: A New Coherent Risk Measure. Journal of Optimization Theory and Applications, 155(3):1105-1123, 2012a. doi:10.1007/s10957-011-9968-2. 2, 3, 23

A. Ahmadi-Javid. Addendum to: Entropic value-at-risk: A new coherent risk measure. Mar 2012b. 2

A. Ahmadi-Javid and A. Pichler. An analytic study of norms and Banach spaces induced by the entropic value-at-risk. Mathematics and Financial Economics, 11:527-550, 2017. doi:10.1007/s11579-017-0197-9. 9

S. Amari. Alpha -Divergence is Unique, Belonging to Both f-Divergence and Bregman Divergence Classes. IEEE Trans. Inform. Theory, 55(11), November 2009. doi:10.1109/TIT.2009.2030485. 2

P. Artzner, F. Delbaen, J.-M. Eber, and D. Heath. Coherent Measures of Risk. Mathematical Finance, 9: 203-228, 1999. doi:10.1111/1467-9965.00068. 3

F. Bellini and E. Rosazza Gianin. On Haezendonck risk measures. Journal of Banking \& Finance, 32(6): 986-994, 2008. doi:10.1016/j.jbankfin.2007.07.007. 3

L. Boltzmann. Über die Beziehung zwischen dem zweiten Hauptsatze der mechanischen Wärmetheorie und der Wahrscheinlichkeitsrechnung resp. den Sätzen über das Wärmegleichgewicht. Sitzungsberichte der kaiserlichen Akademie der Wissenschaften, 76(3):373-435, 1877. 1

T. Breuer and I. Csiszár. Measuring distribution model risk. Mathematical Finance, 2013a. doi:10.1111/mafi.12050. 3

T. Breuer and I. Csiszár. Systematic stress tests with entropic plausibility constraints. Journal of Banking \& Finance, 37(5):1552-1559, 2013b. doi:10.1016/j.jbankfin.2012.04.013. 3

A. Cichocki and S. Amari. Families of Alpha- Beta- and Gamma- Divergences: Flexible and Robust Measures of Similarities. Entropy, 12(6):1532-1568, 2010. ISSN 1099-4300. doi:10.3390/e12061532. 2

T. M. Cover and J. A. Thomas. Elements of Information Theory. Wiley, 2006. 2

F. Delbaen. Coherent risk measures on general probability spaces. In Essays in Honour of Dieter Sondermann, pages 1-37. Springer-Verlag, Berlin, 2002. 4, 7

D. Dentcheva, S. Penev, and A. Ruszczyński. Kusuoka representation of higher order dual risk measures. Annals of Operations Research, 181:325-335, 2010. doi:10.1007/s10479-010-0747-5. 3, 7, 9, 10 
H. Föllmer and T. Knispel. Entropic risk measures: Coherence vs. convexity, model ambiguity and robust large deviations. Stochastics and Dynamics, 11(02n03):333-351,2011. doi:10.1142/S0219493711003334. 3

R. M. Kovacevic and T. Breuer. Multiperiod maximum loss is time unit invariant. SpringerPlus, 5(1):1336, Aug 2016. ISSN 2193-1801. doi:10.1186/s40064-016-2959-x. URL https://doi.org/10.1186/s40064-016-2959-x. 3

S. Kusuoka. On law invariant coherent risk measures. In Advances in mathematical economics, volume 3, chapter 4, pages 83-95. Springer, 2001. doi:10.1007/978-4-431-67891-5. 22

F. Liese and I. Vajda. On Divergences and Informations in Statistics and Information Theory. IEEE Transactions on Information Theory, 52(10), 2006. doi:10.1109/TIT.2006.881731. 2

W. Ogryczak and A. Ruszczyński. Dual stochastic dominance and related mean-risk models. SIAM Journal on Optimization, 13(1):60-78, 2002. doi:10.1137/S1052623400375075. 10

A. Pichler. A quantitative comparison of risk measures. Annals of Operations Research, 2017. doi:10.1007/s10479-017-2397-3. 10

C. E. Shannon. A Mathematical Theory of Communication. Bell System Technical Journal, 27, 1948. 1

T. van Erven and P. Harremos. Rényi Divergence and Kullback-Leibler Divergence. IEEE Transactions on Information Theory, 60:3797 - 3820, 2014. doi:10.1109/TIT.2014.2320500. 2

\section{A Appendix}

We give a proof of Theorem 20. For this we recall the following result first.

Theorem 34 (Envelope Theorem). Let $f(x, q)$ be a continuously differentiable function with $x \in \mathbb{R}$ and $q \in \mathbb{R}$. Assume that the parametric problem

$$
v(q):=\max _{x \in \mathbb{R}} f(x, q),
$$

admits a continuously differentiable solution $x^{*}(q)$. Then the optimal value function $v(q)=f\left(x^{*}(q), q\right)$ of $f$ has the derivative

$$
\frac{\mathrm{d} v}{\mathrm{~d} q}(q)=\frac{\mathrm{d} f}{\mathrm{~d} q}\left(x^{*}(q), q\right) .
$$

Before we give a proof of Theorem 20 we show that $\mathrm{EV} @ \mathrm{R}_{\alpha}^{p}(Y)$ is convex in its dual order.

Lemma 35 (Convexity of the Entropic Value-at-Risk). For $1<p_{0}, p_{1}$ and $0 \leq \lambda \leq 1$ define $p_{\lambda}^{\prime}:=$ $(1-\lambda) p_{0}^{\prime}+\lambda p_{1}^{\prime}$, where $p_{0}^{\prime}\left(p_{1}^{\prime}\right.$, resp. $)$ is the Hölder conjugate exponent of $p_{0}\left(p_{1}\right.$, resp.). Then, for $Y \in L^{\infty}$, $\alpha \in(0,1)$ and $p_{\lambda}:=\frac{p_{\lambda}^{\prime}}{p_{\lambda}^{\prime}-1}$ we have that

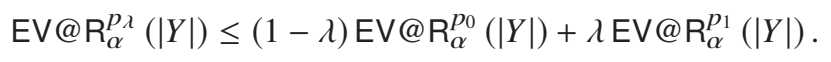

This means the Entropic Value-at-Risk is convex in its conjugate order. 
Remark 36. The following proof of the preceding lemma is rather technical. For this we describe the procedure in brief first. Using the envelope theorem we can calculate the $p^{\prime}$-derivative of the infimum representation (16) at the optimal point. Using the relationship between the optimizer of the infimum and supremum representations we derive a more useful formula for this derivative. We conclude the proof by

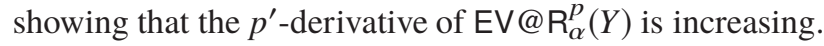

The next lemma shows that the optimizer of the infimum representation (16) of $\mathrm{EV} @ \mathrm{R}_{\alpha}^{p}(Y)$ is nondecreasing. The main result then follows as a simple corollary.

Proof of Lemma 35. Let $\alpha \in(0,1), \beta=\frac{1}{1-\alpha}$ and $Y \in L^{\infty}$. Without loss of generality we may restrict ourselves to $Y>0$ and further suppose that $Y$ is not constant. We apply the envelope theorem to the infimum representation (16) of the Entropic Value-at-Risk (see Theorem 13). The product rule yields

$$
\frac{\mathrm{d}}{\mathrm{d} p^{\prime}} \mathrm{EV} @ \mathrm{R}_{\alpha}^{p}(Y)=\left(\frac{\mathrm{d}}{\mathrm{d} p^{\prime}} \beta^{\frac{p^{\prime}-1}{p^{\prime}}}\right)\left\|(Y-x)_{+}\right\|_{\frac{p^{\prime}}{p^{\prime}-1}}+\left.\beta^{\frac{p^{\prime}-1}{p^{\prime}}} \frac{\mathrm{d}}{\mathrm{d} p^{\prime}}\left\|(Y-x)_{+}\right\|_{\frac{p^{\prime}}{p^{\prime}-1}}\right|_{x=x^{*}\left(p^{\prime}\right)}
$$

where $p^{\prime}$ is the Hölder conjugate of $p>1$. For the remainder of the proof we set $x=x^{*}\left(p^{\prime}\right)$ as it is clear which $p^{\prime}$ is considered. We now detail the above derivative as

$$
\frac{\mathrm{d}}{\mathrm{d} p^{\prime}} \beta^{\frac{p^{\prime}-1}{p^{\prime}}}=\left(\frac{1}{p^{\prime}}\right)^{2} \beta^{\frac{p^{\prime}-1}{p^{\prime}}} \log \beta
$$

and

$$
\frac{\mathrm{d}}{\mathrm{d} p^{\prime}}\left\|(Y-x)_{+}\right\|_{\frac{p^{\prime}}{p^{\prime}-1}}=\left\|(Y-x)_{+}\right\|_{\frac{p^{\prime}}{p^{\prime}-1}} \cdot\left(\frac{1}{p^{\prime^{2}}} \log \mathbb{E}(Y-x)_{+}^{\frac{p^{\prime}}{p^{\prime}-1}}-\frac{1}{\left(p^{\prime}-1\right) p^{\prime}} \frac{\mathbb{E}(Y-x)_{+}^{\frac{p^{\prime}}{p^{\prime}-1}} \log (Y-x)_{+}}{\mathbb{E}(Y-x)_{+}^{\frac{p^{\prime}}{p^{\prime}-1}}}\right) .
$$

Therefore the derivative of EV@ $\mathrm{R}_{\alpha}^{p}$ rewrites as

$$
\begin{aligned}
\frac{\mathrm{d}}{\mathrm{d} p^{\prime}} \mathrm{EV} @ \mathrm{R}_{\alpha}^{p}(Y)= & \beta^{\frac{p^{\prime}-1}{p^{\prime}}}\left\|(Y-x)_{+}\right\|_{\frac{p^{\prime}}{p^{\prime}-1}} \cdot \\
& \left(\frac{1}{p^{\prime^{2}}}\left(\log \beta+\log \mathbb{E}(Y-x)_{+}^{\frac{p^{\prime}}{p^{\prime}-1}}\right)-\frac{1}{\left(p^{\prime}-1\right) p^{\prime}} \cdot \frac{\mathbb{E}(Y-x)_{+}^{\frac{p^{\prime}}{p^{\prime}-1}} \log (Y-x)_{+}}{\mathbb{E}(Y-x)_{+}^{\frac{p^{\prime}}{p^{\prime}-1}}}\right) .
\end{aligned}
$$

We continue by rewriting the last factor. To this end it is useful to consider the maximizing densities of the supremum representation of EV@R, cf. (18),

$$
Z=Z^{*}\left(p^{\prime}\right)=\frac{\left(Y-x^{*}\left(p^{\prime}\right)\right)_{+}^{\frac{1}{p^{\prime}-1}}}{\mathbb{E}\left(Y-x^{*}\left(p^{\prime}\right)\right)_{+}^{\frac{1}{p^{\prime}-1}}}
$$

from which the identity

$$
\mathbb{E}(Y-x)_{+}^{\frac{p^{\prime}}{p^{\prime}-1}}=\mathbb{E} Z^{p^{\prime}} \cdot\left(\mathbb{E}(Y-x)_{+}^{\frac{1}{p^{\prime}-1}}\right)^{p^{\prime}}
$$


follows. Furthermore, by the convexity of the set of feasible densities in (11), each optimal $Z=Z^{*}\left(p^{\prime}\right)$ satisfies the identity $\frac{1}{p^{\prime}-1} \log \mathbb{E} Z^{p^{\prime}}=\log \beta$. For the second factor of (29) we have

$$
\frac{1}{p^{\prime 2}}\left(\log \beta+\log \mathbb{E}(Y-x)_{+}^{\frac{p^{\prime}}{p^{\prime}-1}}\right)=\frac{1}{p^{\prime}}\left(\log \beta+\log \mathbb{E}(Y-x)_{+}^{\frac{1}{p^{\prime}-1}}\right)
$$

where we have used (31) and, by employing (30),

$$
\frac{\mathbb{E}(Y-x)_{+}^{\frac{p^{\prime}}{p^{\prime}-1}} \log (Y-x)_{+}}{\mathbb{E}(Y-x)_{+}^{\frac{p^{\prime}}{p^{\prime}-1}}}=\frac{\left(p^{\prime}-1\right) \mathbb{E} Z^{p^{\prime}} \log Z}{\beta^{p^{\prime}-1}}+\left(p^{\prime}-1\right) \log \mathbb{E}(Y-x)_{+}^{\frac{1}{p^{\prime}-1}} .
$$

In conclusion, the factor $\frac{1}{p^{\prime^{2}}}\left(\log \beta+\log \mathbb{E}(Y-x)_{+}^{\frac{p^{\prime}}{p^{p^{\prime}}-1}}\right)-\frac{1}{\left(p^{\prime}-1\right) p^{\prime}} \cdot \frac{\mathbb{E}(Y-x)_{+}^{\frac{p^{\prime}}{p^{\prime}-1}} \log (Y-x)}{\mathbb{E}(Y-x)_{+}^{\frac{p^{\prime}}{p^{\prime}-1}}}$ in (29) is (cf. (32) and (33))

$$
\frac{1}{p^{\prime}}\left(\log \beta+\log \mathbb{E}(Y-x)_{+}^{\frac{1}{p^{\prime}-1}}\right)-\frac{\mathbb{E} Z^{p^{\prime}} \log Z}{p^{\prime} \beta^{p^{\prime}-1}}-\frac{1}{p^{\prime}} \log \mathbb{E}(Y-x)_{+}^{\frac{1}{p^{\prime}-1}},
$$

which simplifies to

$$
\frac{1}{p^{\prime}} \log \beta-\frac{\mathbb{E} Z^{p^{\prime}} \log Z}{p^{\prime} \beta^{p^{\prime}-1}} .
$$

From the previous considerations we now have the desired formula for the $p^{\prime}$-derivative of $\operatorname{EV} @ \mathrm{R}_{\alpha}^{p}(Y)$ :

$$
\frac{\mathrm{d}}{\mathrm{d} p^{\prime}} \mathrm{EV} @ \mathrm{R}_{\alpha}^{p}(Y)=\beta^{\frac{p^{\prime}-1}{p^{\prime}}}\left\|(Y-x)_{+}\right\|_{\frac{p^{\prime}}{p^{\prime}-1}}\left(\frac{1}{p^{\prime}} \log \beta-\frac{\mathbb{E} Z^{p^{\prime}} \log Z}{p^{\prime} \beta^{p^{\prime}-1}}\right) .
$$

It remains to be seen that this derivative is increasing in $p^{\prime}$, which is equivalent to showing that

$$
p^{\prime} \mapsto \beta^{\prime} \frac{p^{\prime}-1}{p^{\prime}}\left\|(Y-x)_{+}\right\|_{\frac{p^{\prime}}{p^{\prime}-1}}\left(\frac{\mathbb{E} Z^{p^{\prime}} \log Z}{p^{\prime} \beta^{p^{\prime}-1}}-\frac{1}{p^{\prime}} \log \beta\right)
$$

is decreasing. We first show that $p^{\prime} \mapsto \beta^{\prime} \frac{p^{\prime}-1}{p^{\prime}}\left\|(Y-x)_{+}\right\|_{\frac{p^{\prime}}{p^{\prime}-1}}$ is decreasing. From Theorem 19 we know that $p^{\prime} \mapsto \mathrm{EV} @ \mathrm{R}_{\alpha}^{p}(Y)$ is decreasing and hence for the optimizer $x$ of $\operatorname{EV} @ \mathrm{R}_{\alpha}^{p}(Y)$ we have

$$
\frac{\mathrm{d}}{\mathrm{d} p^{\prime}} \mathrm{EV} @ \mathrm{R}_{\alpha}^{p}(Y)=\frac{\mathrm{d}}{\mathrm{d} p^{\prime}} \beta^{\prime \frac{p^{\prime}-1}{p^{\prime}}}\left\|(Y-x)_{+}\right\|_{\frac{p^{\prime}}{p^{\prime}-1}}<0
$$

and thus $p^{\prime} \mapsto \beta^{\prime \frac{p^{\prime}-1}{p^{\prime}}}\left\|(Y-x)_{+}\right\|_{\frac{p^{\prime}}{p^{\prime}-1}}$ is decreasing.

It remains to show that

$$
p^{\prime} \mapsto \frac{\mathbb{E} Z^{p^{\prime}} \log Z}{p^{\prime} \beta^{p^{\prime}-1}}-\frac{1}{p^{\prime}} \log \beta
$$

is decreasing. Note that $\mathbb{E} Z^{*}\left(p^{\prime}\right)^{p^{\prime}} \log Z^{*}\left(p^{\prime}\right) \leq \mathbb{E} Z^{*}\left(p^{\prime}\right)^{p^{\prime}+1} \leq \mathbb{E} C(Y) \cdot\left(Z^{*}\left(p^{\prime}+1\right)\right)^{p^{\prime}+1}=C(Y) \cdot \beta^{p^{\prime}}$ where $C(Y) \geq 1$ is a constant only depending on $Y$. Then

$$
\frac{\mathbb{E} Z^{p^{\prime}} \log Z}{p^{\prime} \beta^{p^{\prime}-1}}-\frac{1}{p^{\prime}} \log \beta \leq \frac{C(Y) \beta^{p^{\prime}}-\beta^{p^{\prime}-1} \log \beta}{p^{\prime} \beta^{p^{\prime}-1}} \leq \frac{C(Y, \beta)}{p^{\prime}},
$$


where $C(Y, \beta)>0$ is a constant only depending on $Y$ and $\beta$ and thus we conclude that (cf. (34))

$$
\frac{\mathrm{d}}{\mathrm{d} p^{\prime}} \mathrm{EV} @ \mathrm{R}_{\alpha}^{p}(Y)=\beta^{\frac{p^{\prime}-1}{p^{\prime}}}\left\|(Y-x)_{+}\right\|_{\frac{p^{\prime}}{p^{\prime}-1}}\left(\frac{1}{p^{\prime}} \log \beta-\frac{\mathbb{E} Z^{p^{\prime}} \log Z}{p^{\prime} \beta^{p^{\prime}-1}}\right)
$$

is increasing in $p^{\prime}$, which is equivalent to the convexity of $p^{\prime} \mapsto \mathrm{EV} @ \mathrm{R}_{\alpha}^{p}$.

Before we give the proof of the main Theorem 20 we analyze the optimizer of the infimum representation of EV@R.

Lemma 37. Let $\alpha \in(0,1), \beta=\frac{1}{1-\alpha}$ and $Y \in L^{\infty}$. As usual for $p>1$ we set $p^{\prime}=\frac{p}{p-1}$. Consider the infimum representation of $\mathrm{EV} @ \mathrm{R}_{\alpha}^{p}(Y)=\inf _{x \in \mathbb{R}} x+\beta^{\frac{1}{p}}\left\|(Y-x)_{+}\right\|_{p}$ with optimizer $x^{*}$. Then the mapping $p^{\prime} \mapsto x^{*}\left(p^{\prime}\right)$ is nondecreasing.

Proof. We may restrict ourselves to $Y>0$ and recall that the derivative of $\operatorname{EV}_{0} \mathrm{R}_{\alpha}^{p}(Y)$ with respect to $x$ is given by

$$
1-\beta^{\frac{1}{p}}\left(\mathbb{E}\left(Y-x^{*}\left(p^{\prime}\right)\right)_{+}^{p}\right)^{\frac{1}{p}-1} \mathbb{E}\left(Y-x^{*}\left(p^{\prime}\right)\right)_{+}^{p-1}
$$

whenever the derivative exists. It follows that $p^{\prime} \mapsto x^{*}\left(p^{\prime}\right)$ is nondecreasing in $p^{\prime}$ if and only if $1-$ $\beta^{\frac{p^{\prime}-1}{p^{\prime}}}\left(\mathbb{E}\left(Y-x^{*}\left(p_{0}^{\prime}\right)\right)_{+}^{\frac{p^{\prime}}{p^{\prime}-1}}\right)^{-\frac{1}{p^{\prime}}} \mathbb{E}\left(Y-x^{*}\left(p_{0}^{\prime}\right)\right)_{+}^{\frac{1}{p^{\prime^{\prime}-1}}}<0$ for all $p^{\prime}>p_{0}^{\prime}$. By convexity of (16) there is a unique optimal $x^{*}\left(p^{\prime}\right)$ for any $p^{\prime}>1$. Furthermore the optimizer $x^{*}\left(p^{\prime}\right)$ is characterized by

$$
\beta\left(\frac{\left\|(Y-x)_{+}\right\|_{\frac{1}{p^{\prime}-1}}}{\left\|(Y-x)_{+}\right\|_{\frac{p^{\prime}}{p^{\prime}-1}}}\right)^{\frac{p^{\prime}}{\left(p^{\prime}-1\right)^{2}}}=1
$$

It is enough to verify the that $p^{\prime} \mapsto\left(\frac{\left\|(Y-x)_{+}\right\| \frac{1}{p^{\prime}-1}}{\left\|(Y-x)_{+}\right\| \frac{p^{\prime}}{p^{\prime}-1}}\right)^{\frac{p^{\prime}}{\left(p^{\prime}-1\right)^{2}}}$ is nondecreasing for fixed $x<\operatorname{ess} \sup Y$. As the logarithm is a strictly increasing function, we may consider the mapping $p^{\prime} \mapsto \log \left(\frac{\left\|(Y-x)_{+}\right\| \frac{1}{\frac{1}{p^{\prime}-1}}}{\left\|(Y-x)_{+}\right\| \frac{p^{\prime}}{p^{\prime}-1}}\right)^{\frac{p^{\prime}}{\left(p^{\prime}-1\right)^{2}}}$ with $p^{\prime}$-derivative

$$
\left(\frac{\mathrm{d}}{\mathrm{d} p^{\prime}} \frac{p^{\prime}}{\left(p^{\prime}-1\right)^{2}}\right) \cdot \log \frac{\left\|(Y-x)_{+}\right\|_{\frac{1}{p^{\prime}-1}}}{\left\|(Y-x)_{+}\right\|_{\frac{p^{\prime}}{p^{\prime}-1}}}+\frac{p^{\prime}}{\left(p^{\prime}-1\right)^{2}} \cdot \frac{\mathrm{d}}{\mathrm{d} p^{\prime}} \log \frac{\left\|(Y-x)_{+}\right\|_{\frac{1}{p^{\prime}-1}}}{\left\|(Y-x)_{+}\right\|_{\frac{p^{\prime}}{p^{\prime}-1}}} .
$$

By monotonicity of the $p$-Norms and by logarithmic convexity of $p^{\prime} \mapsto\|\cdot\|_{p}$ we may conclude that

$$
\frac{\mathrm{d}}{\mathrm{d} p^{\prime}} \log \left(\frac{\left\|(Y-x)_{+}\right\|_{p-1}}{\left\|(Y-x)_{+}\right\|_{p}}\right)^{p(p-1)}>0
$$

It follows immediately that $p^{\prime} \mapsto 1-\beta^{\frac{1}{p}}\left(\mathbb{E}(Y-x)_{+}^{p}\right)^{\frac{1}{p}-1} \mathbb{E}(Y-x)_{+}^{p-1}$ is decreasing and thus

$$
1-\beta^{\frac{1}{p}}\left(\mathbb{E}\left(Y-x^{*}\left(p_{0}^{\prime}\right)\right)_{+}^{p}\right)^{\frac{1}{p}-1} \mathbb{E}\left(Y-x^{*}\left(p_{0}^{\prime}\right)\right)_{+}^{p-1}<0
$$

for all $p^{\prime}>p_{0}^{\prime}$. 
The proof of Theorem 20 now follows as a simple corollary of the preceding 2 lemmas.

Proof of Theorem 20. Suppose the assumptions of Lemma 35 are satisfied. With out loss of generality we may assume that $Y>0$ and that $Y$ is not constant. We then consider the derivative with respect to $p^{\prime}$ using the envelope theorem and obtain

$$
\frac{\mathrm{d}}{\mathrm{d} p^{\prime}} \log \mathrm{EV} @ \mathrm{R}_{\alpha}^{p}(Y)=\frac{\beta^{\frac{p^{\prime}-1}{p^{\prime}}}\left\|\left(Y-x^{*}\left(p^{\prime}\right)\right)_{+}\right\|_{\frac{p^{\prime}}{p^{\prime}-1}}\left(\frac{1}{p^{\prime}} \log \beta-\frac{\mathbb{E} Z^{p^{\prime}} \log Z}{p^{\prime} \beta^{p^{\prime}-1}}\right)}{x^{*}\left(p^{\prime}\right)+\beta^{\frac{p^{\prime}-1}{p^{\prime}}}\left\|\left(Y-x^{*}\left(p^{\prime}\right)\right)_{+}\right\|_{\frac{p^{\prime}}{p^{\prime}-1}}} .
$$

We define $C=x^{*} \cdot\left(\beta^{\frac{p^{\prime}-1}{p^{\prime}}}\left\|\left(Y-x^{*}\right)_{+}\right\|_{\frac{p^{\prime}}{p^{\prime}-1}}\right)^{-1}+1>0$, from which it follows that

$$
\frac{\mathrm{d}}{\mathrm{d} p^{\prime}} \log \mathrm{EV} @ \mathrm{R}_{\alpha}^{p}(Y)=\frac{\left(\frac{1}{p^{\prime}} \log \beta-\frac{\mathbb{E} Z^{p^{\prime}} \log Z}{p^{\prime} \beta^{p^{\prime}-1}}\right)}{C} .
$$

It remains to show that $p^{\prime} \mapsto C\left(p^{\prime}\right)$ is increasing. Since $p^{\prime} \mapsto x^{*}$ is increasing it suffices to show that $x^{*} \mapsto C\left(x^{*}\right)$ is increasing. To see this we differentiate $C\left(x^{*}\left(p^{\prime}\right)\right)$ with respect to $x$,

$$
\begin{aligned}
\frac{\mathrm{d}}{\mathrm{d} x} C\left(x^{*}\left(p^{\prime}\right)\right) & =\beta^{\frac{p^{\prime}-1}{p^{\prime}}}\left\|\left(Y-x^{*}\right)_{+}\right\|_{\frac{p^{\prime}}{p^{\prime}-1}}+x^{*}\left(p^{\prime}\right) \cdot\left(\beta^{\frac{p^{\prime}-1}{p^{\prime}}}\left(\mathbb{E}\left(Y-x^{*}\right)_{+}^{p}\right)^{\frac{1}{p}-1} \mathbb{E}\left(Y-x^{*}\right)_{+}^{p-1}\right) \\
& =\beta^{\frac{p^{\prime}-1}{p^{\prime}}}\left\|\left(Y-x^{*}\right)_{+}\right\|_{\frac{p^{\prime}}{p^{\prime}-1}}+x^{*}\left(p^{\prime}\right) \geq 0 .
\end{aligned}
$$

We conclude that $p^{\prime} \mapsto C\left(Y, \alpha, p^{\prime}\right)$ is increasing and by the proof of Lemma 35 it now follows that

$$
p^{\prime} \mapsto \frac{\left(\frac{\mathbb{E} Z^{p^{\prime}} \log Z}{p^{\prime} \beta^{p^{\prime}-1}}-\frac{1}{p^{\prime}} \log \beta\right)}{C\left(Y, \alpha, p^{\prime}\right)}
$$

is decreasing which concludes the assertion. 